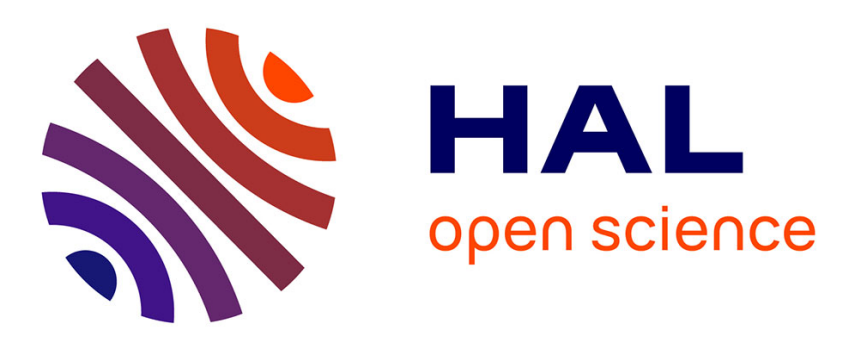

\title{
La réduction des inégalités sociales devant l'école depuis le début du siècle
}

\author{
Claude Thélot, Louis-André Vallet
}

\section{To cite this version:}

Claude Thélot, Louis-André Vallet. La réduction des inégalités sociales devant l'école depuis le début du siècle. Economie et Statistique / Economics and Statistics, 2000, 334, pp.3-32. hal-03455442

HAL Id: hal-03455442

https://hal.science/hal-03455442

Submitted on 29 Nov 2021

HAL is a multi-disciplinary open access archive for the deposit and dissemination of scientific research documents, whether they are published or not. The documents may come from teaching and research institutions in France or abroad, or from public or private research centers.
L'archive ouverte pluridisciplinaire HAL, est destinée au dépôt et à la diffusion de documents scientifiques de niveau recherche, publiés ou non, émanant des établissements d'enseignement et de recherche français ou étrangers, des laboratoires publics ou privés. 
La réduction des inégalités sociales devant l'école depuis le début du siècle

Monsieur Claude Thélot, Monsieur Louis-André Vallet

\section{Citer ce document / Cite this document :}

Thélot Claude, Vallet Louis-André. La réduction des inégalités sociales devant l'école depuis le début du siècle. In: Economie et statistique, $n^{\circ} 334,2000$. pp. 3-32;

http://www.persee.fr/doc/estat_0336-1454_2000_num_334_1_7526

Document généré le 23/05/2016 


\title{
Zusammenfassung
}

Die Schulpflicht, die Verallgemeinerung des Unterrichts der Sekundarstufe sowie schließlich die Öffnung des Hochschulunterrichts verdeutlichen das Bestreben, die Schul-und Universitätsausbildung Bevölkerungsschichten zugänglich zu machen, denen sie früher verschlossen war, und die sozialen Ungleichheiten im Hinblick auf die Schule abzubauen. Die Verwirklichung dieses Ziels bewerten heißt, den jeweiligen Anteil messen, den drei Faktoren an der Entwicklung des schulischen Werdegangs der Jugendlichen entsprechend ihrer sozialen Herkunft haben: grundlegender Wandel in der sozialen Struktur, allgemeine Verlängerung der Schulzeit und Veränderung der Beziehung zwischen Herkunft und Diplom. Mit der Analyse des schulischen Werdegangs entsprechend der sozialen Herkunft für die zwischen 1908 und 1972 geborenen Generationen kann man die Rolle, die diese Faktoren seit nahezu einem Jahrhundert spielen, sowie ihre relative Bedeutung bestimmen. Die allgemeine Verlängerung der Schulzeit ist der Hauptfaktor dieser Entwicklung: sie erklärt drei Viertel des Unterschieds, der zwischen den extremen Generationen bei den schulischen Werdegängen entsprechend der sozialen Herkunft bestehen. Über diesen Effekt hinaus hat eine qualitative Demokratisierung nicht minder erhebliche Auswirkungen: auf die Schwächung der Beziehung zwischen sozialer Herkunft und Diplom ist rund ein Siebtel des Unterschieds zurückzuführen. In den fünfziger und siebziger Jahren und danach in jüngster Zeit hat sich diese Demokratisierung voll ausgewirkt. Von ihr profitierten mehr die Mädchen als die Jungen. Abgenommen hat auch die Beziehung zwischen kultureller Herkunft und Diplom, allerdings langsamer als die Beziehung zwischen sozialer Herkunft und Diplom.

Insgesamt sind die sozialen Ungleichheiten im Hinblick auf die Schule auch heute noch sehr groß. Seit einigen Jahrzehnten haben sie allerdings abgenommen, nicht nur aufgrund der allgemeinen Verlängerung der Schulzeit, sondern auch darüber hinaus in qualitativer Hinsicht.

\begin{abstract}
Compulsory education, the spread of secondary education and the opening up of higher education show the will to make school and university education accessible to those social groups for whom it was previously unattainable and to reduce the social inequalities in education. Assessing the attainment of this goal consists in measuring the respective proportion of three factors in the development of the educational paths of young people, based on their social origins: the change in the social structure, the general lengthening of education, and the change in the link between social origins and qualifications. An analysis of educational paths by social origins for the generations born from 1908 to 1972 describes the role of these factors and their relative importance over nearly a century. The general lengthening of education is the main factor in this development. It explains three-quarters of the difference in educational paths based on social origins between the two extreme generations. Qualitative democratisation also has an appreciable impact. The weakening of the link between social origins and qualifications is responsible for approximately one-seventh of the deviation. This democratisation took full effect in the 1950s and 1960s and again just recently. It has been more to the advantage of girls than boys. The link between cultural origin and qualifications has also weakened, but at a slower rate than that between social origins and qualifications. Overall, social inequalities in education are still strong today. However, they have diminished over the last few decades with the widespread lengthening of education and qualitative improvements.
\end{abstract}

\section{Résumé}

L'instruction obligatoire, la généralisation de l'enseignement secondaire, et enfin l'ouverture de l'enseignement supérieur illustrent la volonté de rendre accessible la formation scolaire et universitaire à des milieux auxquels elle était autrefois fermée et de réduire les inégalités sociales devant l'école. Apprécier la réalisation de cet objectif revient à mesurer la part respective des trois facteurs de l'évolution de la destinée scolaire des jeunes en fonction de leur origine sociale: la mutation de la structure sociale, l'allongement général de la scolarisation, et la modification du lien entre milieu d'origine et diplôme. L'analyse de la destinée scolaire selon l'origine sociale, pour les générations nées entre 1908 et 1972, permet de préciser le rôle de ces facteurs depuis près d'un siècle, ainsi que leur importance relative. L'allongement général des études est le facteur principal 
de l'évolution: il explique les trois quarts de la différence des destinées scolaires selon l'origine sociale, entre les générations extrêmes. Au-delà de cet effet, une démocratisation qualitative n'en a pas moins un impact appréciable: l'affaiblissement du lien entre origine sociale et diplôme est responsable d'un septième environ de l'écart. C'est dans les années 50 et 60 , puis tout récemment, que cette démocratisation a joué à plein. Elle s'est davantage exercée au profit des filles que des garçons. Le lien entre origine culturelle et diplôme s'est lui aussi affaibli, mais à un rythme plus lent que le lien entre origine sociale et diplôme. Au total, les inégalités sociales devant l'école demeurent aujourd'hui fortes. Elles ont cependant diminué depuis quelques décennies, non seulement du fait de l'allongement général des études, mais aussi audelà, de façon qualitative.

\section{Resumen}

La instrucción obligatoria, la generalización de la enseñanza secundaria, y en fin la apertura de la enseñanza superior ponen de manifiesto la voluntad de hacer que sea accesible la formación escolar y universitaria a unos medios a que antes no podían disfrutar de ella, y de reducir las desigualdades sociales ante la escuela. Apreciar el cumplimiento de este objetivo equivale a medir la proporción de cada uno de los tres factores de evolución del destino escolar de los jóvenes según su origen social: el cambio de la estructura social, el alargamiento general de la escolarización, y la modificación de la relación entre medio de origen y diploma.

El análisis del destino escolar según el origen social, para aquellas generaciones nacidas entre 1908 y 1972, permite precisar el papel de estos factores desde hace casi un siglo, y su peso relativo. El alargamiento general de los estudios es el factor principal de la evolución: a éste se deben los tres cuartos de la diferencia de los destinos escolares según el origen social, entre las generaciones extremas. Además de este efecto, una democratización cualitativa tiene también un impacto apreciable: la distensión de la relación entre origen social y diploma es responsable de una séptima parte de la diferencia. Tal democratización ha culminado en los años cincuenta y sesenta y hace muy poco. Ésta ha favorecido más a las chicas que a los chicos. La relación entre origen cultural y diploma también se ha aflojado pero a un ritmo más lento que la relación entre origen social y diploma.

En resumen, las desigualdades sociales ante la escuela son todavía muy fuertes. Sin embargo, se han reducido desde hace unos decenios no sólo debido al alargamiento general de los estudios, sino también de manera cualitativa. 


\title{
La réduction des inégalités sociales devant l'école depuis le début du siècle
}

\author{
Claude Thélot et Louis-André Vallet*
}

L'instruction obligatoire, la généralisation de l'enseignement secondaire, et enfin l'ouverture de l'enseignement supérieur illustrent la volonté de rendre accessible la formation scolaire et universitaire à des milieux auxquels elle était autrefois fermée et de réduire les inégalités sociales devant l'école. Apprécier la réalisation de cet objectif revient à mesurer la part respective des trois facteurs de l'évolution de la destinée scolaire des jeunes en fonction de leur origine sociale : la mutation de la structure sociale, l'allongement général de la scolarisation, et la modification du lien entre milieu d'origine et diplôme.

L'analyse de la destinée scolaire selon l'origine sociale, pour les générations nées entre 1908 et 1972, permet de préciser le rôle de ces facteurs depuis près d'un siècle, ainsi que leur importance relative. L'allongement général des études est le facteur principal de l'évolution : il explique les trois quarts de la différence des destinées scolaires selon l'origine sociale, entre les générations extrêmes. Au-delà de cet effet, une démocratisation qualitative n'en a pas moins un impact appréciable : l'affaiblissement du lien entre origine sociale et diplôme est responsable d'un septième environ de l'écart. C'est dans les années 50 et 60 , puis tout récemment, que cette démocratisation a joué à plein. Elle s'est davantage exercée au profit des filles que des garçons. Le lien entre origine culturelle et diplôme s'est lui aussi affaibli, mais à un rythme plus lent que le lien entre origine sociale et diplôme.

Au total, les inégalités sociales devant l'école demeurent aujourd'hui fortes. Elles ont cependant diminué depuis quelques décennies, non seulement du fait de l'allongement général des études, mais aussi au-delà, de façon qualitative.

* Claude Thélot est conseiller maître à la Cour des comptes et Louis-André Vallet est chargé de recherche au Laboratoire d'analyse secondaire et des méthodes appliquées à la sociologie - Institut du longitudinal (CNRS). Tous deux sont aussi membres associés du Laboratoire de sociologie quantitative (Crest - Insee).

Les noms et dates entre parenthèses renvoient à la bibliographie en fin d'article. 
$\mathbf{L}$ 'école a toujours joué dans notre pays un rôle central. Même lorsqu'elle était fréquentée par peu d'enfants, ou peu longtemps, même lorsqu'elle était constituée en filières. À plus forte raison aujourd'hui qu'elle est devenue l'école de masse : instruction obligatoire jusqu'à 13 ans (il y a 120 ans), jusqu'à 16 ans (il y a 40 ans), collège unique (il y a 25 ans), lycée de masse (il y a 12 ans), enseignement supérieur de masse, désormais la durée moyenne de scolarisation (y compris préélémentaire) est de 19 ans, et le rôle structurant de l'école, sa fonction de creuset pour toute une génération se sont de fait accrus. D'où un intérêt, accru lui aussi, pour les inégalités devant le système scolaire, inégalités de toutes natures, et, en particulier, géographiques, entre garçons et filles, entre classes sociales. Réussir l'école de masse, confier à l'école, jusqu'à l'excès, la plupart des fonctions de formation, d'éducation et de socialisation supposent que les inégalités devant l'école ne soient pas trop grandes.

L'égalité des chances devant l'école est une exigence, ou une ambition, assez récente: elle ne date guère que de la Seconde Guerre mondiale, ou même de l'entrée dans la société de consommation, c'est-à-dire du début des années 60. Auparavant, le système scolaire... n'était pas un système : il y avait, assez séparées, même si elles étaient socialement moins étanches que ce qu'on a souvent dit, deux filières : la filière primaire (école communale, puis, éventuellement, certificat d'études primaires, primaire supérieur, brevet, brevet supérieur), la filière secondaire (petite classe de lycée, lycée, baccalauréat, éventuellement études supérieures). Aujourd'hui, ces deux filières ont fusionné, tous les jeunes vont au collège, deux tiers vont au lycée (ou au lycée professionnel), plus de la moitié entrent dans l'enseignement supérieur. Il y a donc eu «massification» de l'école, c'est-à-dire allongement général des études, ou encore accroissement de la scolarisation pour l'ensemble des jeunes, quel que soit leur milieu d'origine. Cette massification peut s'accompagner d'une simple translation des inégalités, les jeunes d'origine sociale modeste accédant à des niveaux de formation inconnus auparavant, mais les écarts de destinée scolaire entre jeunes des différents milieux sociaux demeurant aussi intenses. La massification peut, au contraire, aller de pair avec une réduction de ces écarts, ce qui correspond à un affaiblissement du lien entre origine sociale et destinée scolaire, l'accès aux diplômes les plus élevés s'élargissant davantage pour les jeunes d'origine sociale modeste. Dans ce dernier cas, la démocratisation est plus prononcée et la réduction des écarts entre milieux sociaux est souvent nommée «démocratisation qualitative » (Prost, 1986).

La question de l'égalité des chances devant l'école est alors souvent posée en ces termes : cette massification, évidente et qui n'est pas contestable, s'est-elle accompagnée, en plus, d'une démocratisation qualitative ? C'est à répondre à cette question qu'est consacré cet article. Il retrace l'évolution des inégalités sociales devant l'école depuis les premières décennies du siècle. La question concerne bien sûr l'école, mais pas seulement : puisque, depuis 30 ou 40 ans, la formation et les diplômes jouent un rôle beaucoup plus grand qu'autrefois dans l'accès aux positions sociales, la question a trait aussi à la constitution des élites de la société. Réussir à l'école est devenu quasiment une condition nécessaire pour rallier ces dernières - d'autres voies comme le syndicat, le parti politique, ou même la réussite individuelle répondant au modèle du self made man s'avérant aujourd'hui moins efficaces - et les inégalités sociales devant l'école pèsent davantage sur la formation des élites, leur renouvellement et leur élargissement, ou, au contraire, leur auto-reproduction avec, pour corollaire, leur fermeture sur elles-mêmes.

Le matériau empirique est, pour l'essentiel, constitué des enquêtes de l'Insee qui, depuis 35 ans, permettent de suivre, génération par génération, les diplômes qui ont été obtenus: enquêtes Formation et Qualification Professionnelle (FQP) et enquêtes Emploi (cf. encadré 1). Les générations ont été regroupées, non pas de façon aléatoire ou arbitraire, mais en fonction de l'histoire scolaire et, plus précisément, des politiques scolaires. Les enquêtes, ces regroupements et la nécessité de se limiter à des générations qui ont terminé leurs études conduisent à définir 13 groupes de cinq générations: des personnes nées de 1908 à 1912, générations les plus anciennes, à celles nées de 1968 à 1972, générations les plus récentes ayant aujourd'hui pratiquement achevé leurs études. Pour analyser les dernières évolutions de l'accès au baccalauréat, ces enquêtes sont aussi complétées à l'aide de données, collectées par le ministère de l'Éducation nationale, sur le suivi des élèves au cours de leurs études secondaires. 
Encadré 1

\section{DONNÉES ET NOMENCLATURES}

Cet article utilise les données issues des cinq enquêtes Formation et Qualification Professionnelle conduites en 1964, 1970, 1977, 1985 et 1993 ainsi que des enquêtes Emploi de 1993 et 1997. On a retenu, dans chacune, les Français de naissance, hommes et femmes, âgés d'au moins 25 ans et qui ont précisé la situation professionnelle de leur père (ou, à défaut, de leur tuteur, ou de leur mère dans les enquêtes Emploi) au moment où eux-mêmes achevaient leurs études. Ces enquêtes ont été utilisées pour constituer des tables de destinée scolaire en fonction de l'origine sociale, relatives à 13 groupes de cinq générations (ou générations quinquennales). Afin de disposer d'effectifs aussi nombreux que possible pour chacune de ces 13 générations quinquennales, on a le plus souvent cumulé les échantillons des différentes enquêtes communs à chacune de ces générations. L'effectif étudié est donc variable d'une génération à l'autre (cf. tableau $A$ ).
Pour tenir compte de la forte variation des taux de sondage à l'intérieur d'une même enquête, chacun des 49 tableaux croisant origine sociale et diplôme le plus élevé obtenu a été extrait du fichier d'enquête considéré sur la base des données extrapolées à la population française. Puis, au moyen d'une simple règle de trois, il a été ramené à l'effectif total réellement interrogé qui lui correspond (cf. les effectifs entre parenthèses dans le tableau A). C'est en ce sens que, dans le tableau 1 et pour une case quelconque, nous parlons d'observations « reconstituées». Un tel procédé permet de raisonner sur l'effectif total réellement enquêté - ce qui est nécessaire pour les besoins des tests statistiques sans introduire de biais dans la structure du tableau croisé - ce qui aurait été le cas du fait des taux de sondage différents si, pour chaque case du tableau, on avait retenu l'effectif non pondéré. Ce sont enfin ces tableaux d'observations « reconstituées » qui ont été

Tableau A

Répartition de l'échantillon des enquêtes entre les générations étudiées

\begin{tabular}{|c|c|c|c|c|c|c|c|}
\hline $\begin{array}{l}\text { Enquête } \\
\text { Génération }\end{array}$ & FQP 1964 & FQP 1970 & FQP 1977 & FQP 1985 & FQP 1993 & Emploi 1993 & Emploi 1997 \\
\hline 1908-1912 & & $\begin{array}{l}\text { 58-62 ans } \\
(2304)\end{array}$ & $\begin{array}{c}\text { 65-69 ans } \\
(1273)\end{array}$ & & & & \\
\hline 1913-1917 & & $\begin{array}{c}53-57 \text { ans } \\
(1920)\end{array}$ & $\begin{array}{c}\text { 60-64 ans } \\
(1372)\end{array}$ & & & & \\
\hline $1918-1922$ & $\begin{array}{c}\text { 42-46 ans } \\
\text { (2 219) }\end{array}$ & $\begin{array}{l}\text { 48-52 ans } \\
(2756)\end{array}$ & $\begin{array}{l}\text { 55-59 ans } \\
(2218)\end{array}$ & $\begin{array}{c}\text { 63-67 ans } \\
(1264)\end{array}$ & & & \\
\hline 1923-1927 & $\begin{array}{c}\text { 37-41 ans } \\
\text { (2 635) }\end{array}$ & $\begin{array}{c}\text { 43-47 ans } \\
\text { (3 561) }\end{array}$ & $\begin{array}{c}\text { 50-54 ans } \\
(3361)\end{array}$ & $\begin{array}{c}\text { 58-62 ans } \\
(2 \text { 073) }\end{array}$ & & & \\
\hline 1928-1932 & $\begin{array}{l}\text { 32-36 ans } \\
\text { (2 622) }\end{array}$ & $\begin{array}{c}\text { 38-42 ans } \\
\text { (3 630) }\end{array}$ & $\begin{array}{c}\text { 45-49 ans } \\
\text { (3 477) }\end{array}$ & $\begin{array}{c}\text { 53-57 ans } \\
(2729)\end{array}$ & & & \\
\hline 1933-1937 & $\begin{array}{c}27-31 \text { ans } \\
\text { (2 349) }\end{array}$ & $\begin{array}{c}\text { 33-37 ans } \\
\text { (3 369) }\end{array}$ & $\begin{array}{c}\text { 40-44 ans } \\
\text { (3 351) }\end{array}$ & $\begin{array}{l}\text { 48-52 ans } \\
\text { (2 953) }\end{array}$ & $\begin{array}{c}56-60 \text { ans } \\
(1505)\end{array}$ & $\begin{array}{c}56-60 \text { ans } \\
\text { (8 258) }\end{array}$ & \\
\hline 1938-1942 & & $\begin{array}{l}\text { 28-32 ans } \\
\text { (3 394) }\end{array}$ & $\begin{array}{c}\text { 35-39 ans } \\
(3050)\end{array}$ & $\begin{array}{c}\text { 43-47 ans } \\
\text { (2 813) }\end{array}$ & $\begin{array}{c}51-55 \text { ans } \\
(1437)\end{array}$ & $\begin{array}{c}51-55 \text { ans } \\
(7467)\end{array}$ & $\begin{array}{c}\text { 55-59 ans } \\
(7332)\end{array}$ \\
\hline 1943-1947 & & & $\begin{array}{c}\text { 30-34 ans } \\
(4872)\end{array}$ & $\begin{array}{c}\text { 38-42 ans } \\
\text { (3 767) }\end{array}$ & $\begin{array}{c}46-50 \text { ans } \\
(1850)\end{array}$ & $\begin{array}{c}46-50 \text { ans } \\
(9778)\end{array}$ & $\begin{array}{c}50-54 \text { ans } \\
(9078)\end{array}$ \\
\hline 1948-1952 & & & $\begin{array}{c}25-29 \text { ans } \\
(5131)\end{array}$ & $\begin{array}{c}\text { 33-37 ans } \\
\text { (5 044) }\end{array}$ & $\begin{array}{c}\text { 41-45 ans } \\
(2 \text { 107) }\end{array}$ & $\begin{array}{c}41-45 \text { ans } \\
\text { (12 239) }\end{array}$ & $\begin{array}{c}45-49 \text { ans } \\
(11597)\end{array}$ \\
\hline 1953-1957 & & & & $\begin{array}{c}\text { 28-32 ans } \\
\text { (5 212) }\end{array}$ & $\begin{array}{c}36-40 \text { ans } \\
(2068)\end{array}$ & $\begin{array}{c}36-40 \text { ans } \\
(11928)\end{array}$ & $\begin{array}{c}40-44 \text { ans } \\
(11481)\end{array}$ \\
\hline $1958-1962$ & & & & & $\begin{array}{c}\text { 31-35 ans } \\
(1942)\end{array}$ & $\begin{array}{c}31-35 \text { ans } \\
(11689)\end{array}$ & $\begin{array}{c}\text { 35-39 ans } \\
(11725)\end{array}$ \\
\hline 1963-1967 & & & & & $\begin{array}{c}26-30 \text { ans } \\
(1904)\end{array}$ & $\begin{array}{c}26-30 \text { ans } \\
(11803)\end{array}$ & $\begin{array}{c}\text { 30-34 ans } \\
(11621)\end{array}$ \\
\hline $1968-1972$ & & & & & & & $\begin{array}{c}25-29 \text { ans } \\
(11063)\end{array}$ \\
\hline
\end{tabular}

Lecture : l'âge indiqué est l'âge atteint au cours de l'année d'enquête. Entre parenthèses, on a indiqué l'effectif réel de la génération considérée dans l'échantillon de l'enquête en question.

Champ : individus français de naissance, âgés d'au moins 25 ans, ayant précisé la situation professionnelle de leur père (ou, à défaut, de leur tuteur, ou de leur mère dans le cas de l'enquête Emploi). 
Encadré 1 (suite)

éventuellement cumulés entre les différentes enquêtes pour une même génération quinquennale. La démarche précédente a été suivie pour l'ensemble des deux sexes, mais aussi pour les hommes et les femmes séparément.

Au total, six indicateurs d'origine sociale ont été mis en œuvre. L'indicateur principal d'origine sociale (OS), utilisable dans les sept enquêtes, est fondé sur la catégorie socioprofessionnelle du père, observée en huit postes :

1. Agriculteurs exploitants (CSP 00 ; PCS 11 à 13)

2. Artisans, petits commerçants

(CSP 22, 23 et 27 ; PCS 21 et 22)

3. Gros indépendants et cadres supérieurs

(CSP 21, 26, 30, 33 et 34 ; PCS 23, 31, 33, 35, 37 et 38)

4. Enseignants et assimilés

(CSP 32 et 41 ; PCS 34 et 42 )

5. Cadres moyens (CSP 42 à $44 ;$ PCS 43 à 47)

6. Employés

(CSP 51, 53, 70 à 72, 80 à 82 ; PCS 52 à 56)

7. Contremaîtres et ouvriers qualifiés

(CSP 60, 61, 65 à 67 ; PCS 48, 62, 63 et 65)

8. Ouvriers non qualifiés et agricoles

(CSP 10, 63 et 68 ; PCS 64, 67 à 69).

Les cinq autres indicateurs d'origine sociale ne peuvent être définis qu'à partir des enquêtes $F Q P$ de 1977 , 1985 et 1993. C'est alors la recodification en nomenclature PCS de la première d'entre elles (1977) qui est utilisée et la dernière génération observable est celle de 1963-1967 (car elle a terminé ses études à la date de l'enquête de 1993). Ces cinq indicateurs sont les suivants : catégorie socioprofessionnelle de la mère ou diplôme lorsqu'elle a toujours été inactive (OSM en douze postes), catégorie socioprofessionnelle croisée des deux parents (OSPM en dix postes), catégorie socioprofessionnelle et diplôme le plus élevé du père (OSDIPP en dix postes), catégorie socioprofessionnelle du père et diplôme le plus élevé de la mère (OSDIPM en dix postes), diplôme le plus élevé des deux parents (DIPPM en six postes).

Aussi souvent que possible, c'est-à-dire à l'exception de l'enquête FQP de 1964 et des deux enquêtes Emploi, c'est le diplôme le plus élevé obtenu en formation initiale par le répondant qui a été pris en considération.
La nomenclature de diplômes la plus détaillée comporte sept postes (DIPL7)

1. Aucun diplôme ou non déclaré

2. Certificat d'études primaires

3. Brevet élémentaire, BEPC (sans diplôme technique)

4. CAP, BEP (ou diplôme de niveau équivalent : examen de fin d'apprentissage artisanal, etc.)

5. Baccalauréat (ou diplôme de niveau équivalent : BEI, BEC, BEA, etc.)

6. Diplôme d'une ou deux années après le baccalauréat, diplôme pédagogique, diplôme paramédical ou social

7. Diplôme d'au moins trois années après le baccalauréat.

Des variantes ont aussi été utilisées de façon systématique, en regroupant certaines modalités de DIPL7 :

- DI1 (5 postes), obtenu en regroupant aucun diplôme ou non déclaré et CEP d'une part, les deux niveaux de l'enseignement supérieur d'autre part ;

- DI2 (6 postes), obtenu essentiellement pour les générations 1908-1912 à 1948-1952 en regroupant les deux niveaux de l'enseignement supérieur ;

- DI3 (6 postes), obtenu essentiellement pour les générations 1953-1957 à 1968-1972 en regroupant aucun diplôme ou non déclaré et CEP ;

- DI4 (6 postes), obtenu en combinant DI2 et DI3 selon l'année de naissance, ce qui équivaut à postuler une dévalorisation des diplômes à partir de la génération 1953-1957. La correspondance entre diplômes suivant l'année de naissance est détaillée au tableau $B$.

Pour les individus nés avant 1918, l'enquête $F Q P$ de 1970 ne retenait pas de distinction entre les diplômes de l'enseignement supérieur. L'étude de l'évolution du lien entre catégorie socioprofessionnelle du père et diplôme le plus élevé obtenu dans la nomenclature DIPL7 porte donc au maximum sur un effectif total de 240367 observations : 1273 dans la génération 19081912, 11063 dans la génération 1968-1972, 36118 dans la génération la plus représentée, celle de 19481952. Ces effectifs deviennent respectivement 244591 , 3577,11063 et 36118 dès qu'une nomenclature de diplômes moins fine est adoptée. L'étude de l'évolution du lien entre les autres indicateurs d'origine sociale et le diplôme le plus élevé obtenu porte, quant à elle, sur un effectif total de 66781 observations : 1274 dans la génération 1908-1912, 1904 dans la génération 19631967, et 12283 dans la génération la plus représentée, celle de 1948-1952.

\section{Tableau B}

\section{Correspondance entre diplômes dans l'hypothèse d'une dévalorisation}

\begin{tabular}{|c|c|c|}
\hline Nomenclature DI4 & Naissance entre 1908 et 1952 & Naissance entre 1953 et 1972 \\
\hline 1 & Aucun diplôme & Aucun diplôme ou CEP \\
2 & CEP & Brevet, BEPC \\
3 & CAP, BEP & CAP, BEP \\
4 & Brevet, BEPC & Baccalauréat \\
5 & Baccalauréat & Baccalauréat +1 ou 2 an(s) \\
6 & Supérieur au baccalauréat & Baccalauréat +3 ans et plus
\end{tabular}

Lecture : l'obtention du brevet ou du BEPC dans les générations nées entre 1953 et 1972 est supposée équivalente à l'obtention du certificat d'études primaires dans celles nées entre 1908 et 1952. L'hypothèse de dévalorisation retenue est forte et surestime certainement la dérive réelle des diplômes. 
Encadré 1 (fin)

Le souhait de combiner dans une même analyse des données issues de plusieurs enquêtes a conduit à se poser la question de leur éventuelle hétérogénéité. La distribution des origines sociales d'une même génération est-elle stable au fil des enquêtes ? Celles-ci enregistrent-elles de la même manière les diplômes des individus nés les mêmes années ? L'association statistique entre origine sociale et diplôme varie-t-elle selon l'enquête pour une même génération ? On a procédé de façon systématique à des tests d'homogénéité. En raison de l'importance des échantillons mis en jeu, ils amènent souvent à rejeter l'hypothèse d'homogénéité sans que cela corresponde pourtant à des écarts si amples et $\mathrm{s}$ systématiques qu'ils conduiraient à écarter toute combinaison des différentes enquêtes. Cette conclusion vaut en particulier pour l'analyse de l'association statistique nette des effets de marges, qui constitue l'apport essentiel de cet article. Le graphique de cet encadré illustre ainsi qu'une conclusion sans ambiguïté sur l'évolution en longue période de la force du lien entre origine sociale et diplôme peut être obtenue à partir de la cinquantaine de tableaux initiaux (génération $x$ enquête).
Certaines variations dans les distributions marginales de diplôme et d'origine sociale méritent néanmoins d'être signalées. L'enquête FQP de 1964 s'écarte des autres par un poids excessif des diplômes de niveau CAP : le fait qu'elle n'isole pas la seule formation initiale pourrait en être responsable. De même, peut-être pour la même raison et comparativement aux enquêtes $F Q P$, les enquêtes Emploi surreprésentent légèrement les diplômes de l'enseignement supérieur au détriment du baccalauréat. La légère élévation au fil du temps du niveau de diplôme déclaré (Baudelot, 1989) a aussi été mise en évidence. Du point de vue de l'origine sociale, on relève un effet de nomenclature lié au passage des CSP aux PCS. Cet effet est toutefois moins fort que l'effet du type d'enquête, opposant l'enquête $F Q P$ à l'enquête Emploi. Les enquêtes Emploi surreprésentent en effet la catégorie des employés et sous-représentent celle des contremaîtres et ouvriers qualifiés. Ici encore, ce pourrait être lié au fait qu'en l'absence du père, c'est la position socioprofessionnelle de la mère qui a pu être déclarée alors que ce n'est pas le cas dans les enquêtes FQP.

\section{Lien entre origine sociale (position du père) et diplôme (code détaillé) sous le modèle d'évolution uniforme des rapports de chances (1)}

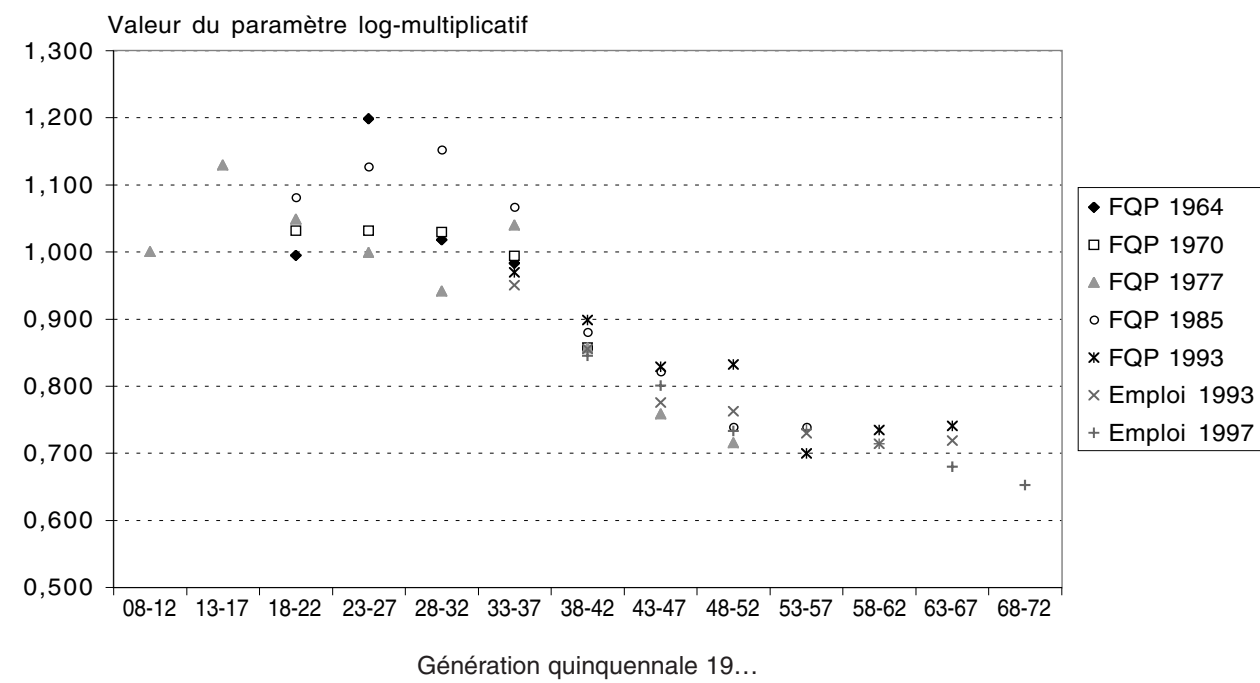

1. L'échantillon considéré ici a un effectif total de 240367 individus. Pour le champ et la source, on se reportera au texte de cet encadré. Le modèle a été estimé simultanément sur les 47 tableaux génération x enquête (cf. tableau A).

Lecture : en supposant stable la valeur des diplômes, le lien entre position du père et diplôme s'affaiblit de 1 (génération 1908-1912, enquête FQP 1977) à 0,653 (génération 1968-1972, enquête Emploi 1997).

\section{Différentes voies pour repérer le milieu d'origine}

Repérer l'origine sociale des membres de ces générations nécessite de faire des choix. Deux seront privilégiés. D’abord, elle sera identifiée par la position sociale du père des personnes, lorsque celles-ci achevaient leurs études, en retenant huit postes : les postes habituels, mais en identifiant, en plus, les enseignants (et assi- milés) et en distinguant ouvriers qualifiés et ouvriers non qualifiés. Ensuite, on combinera la position sociale du père et le diplôme le plus élevé de la mère, en raison de l'importance capitale de cette dernière dans la réussite scolaire des enfants. Complétant ces deux façons de repérer leur origine sociale, l'origine culturelle des personnes sera également utilisée. Elle sera appréhendée au travers du diplôme le plus élevé obtenu par les deux parents. 
L'évolution de l'origine sociale des générations depuis le début du siècle peut être résumée en quelques grandes tendances: effondrement des origines paysannes, baisse des origines indépendantes, augmentation puis diminution des origines ouvrières (la diminution étant plus ancienne et plus prononcée pour les ouvriers non qualifiés), augmentation générale de la part des familles « tertiaires », particulièrement celles d'employés et de cadres moyens, mais aussi d'enseignants dans les quatre dernières générations, c'est-à-dire depuis le milieu des années 50 (cf. graphique I). La combinaison de la position paternelle et du diplôme maternel accuse certains de ces traits : ce sont surtout les familles paysannes où la mère n'avait aucun titre scolaire qui ont disparu et il en est de même pour celles d'indépendants. De façon analogue, le contraste est marqué entre les jeunes issus de familles ouvrières où la mère n'avait pas de diplôme, dont le poids oscille entre $25 \%$ et $30 \%$ (sauf dans la dernière génération (1)) et celles où la mère était diplômée, dont le poids n'a cessé de croître (cf. graphique II). Dans

1. Dans le graphique II où l'origine sociale est repérée par une combinaison entre la position du père et le diplôme de la mère, l'évolution ne porte que sur 12 groupes de générations, le dernier étant constitué de celles nées entre 1963 et 1967. Cela provient du fait que l'information sur le diplôme de la mère n'est disponible que dans les enquêtes FQP. La dernière a été réalisée en 1993, date à laquelle les générations nées entre 1968 et 1972 (le $13^{\circ}$ groupe de l'analyse) n'avaient pas terminé leurs études. Elles les ont achevées, en revanche, lors de l'enquête sur l'emploi de 1997, dernière enquête retenue, d'où leur présence dans le graphique $I$.

Graphique I

\section{Origine sociale (position du père) selon la génération (1)}

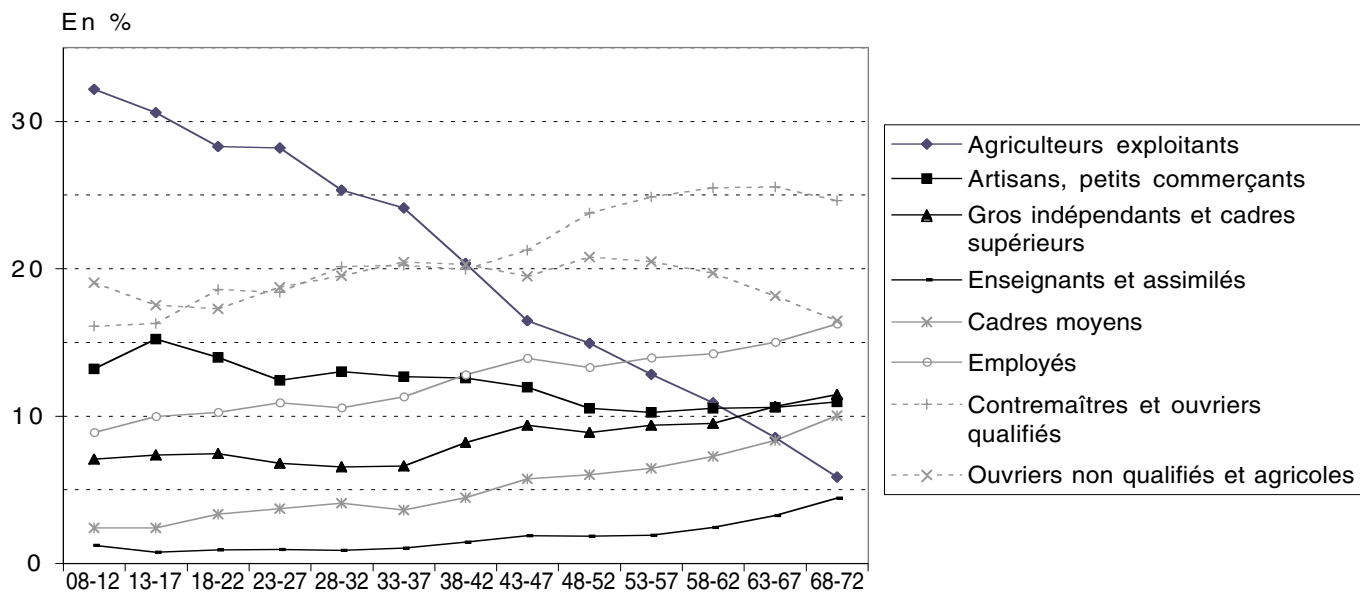

1. L'échantillon considéré ici a un effectif total de 244591 individus. Pour le champ et la source, on se reportera à l'encadré 1 Lecture : environ 32 \% des individus nés entre 1908 et 1912 avaient un père agriculteur exploitant.

Graphique II

\section{Origine sociale (position du père/diplôme de la mère) selon la génération (1)}

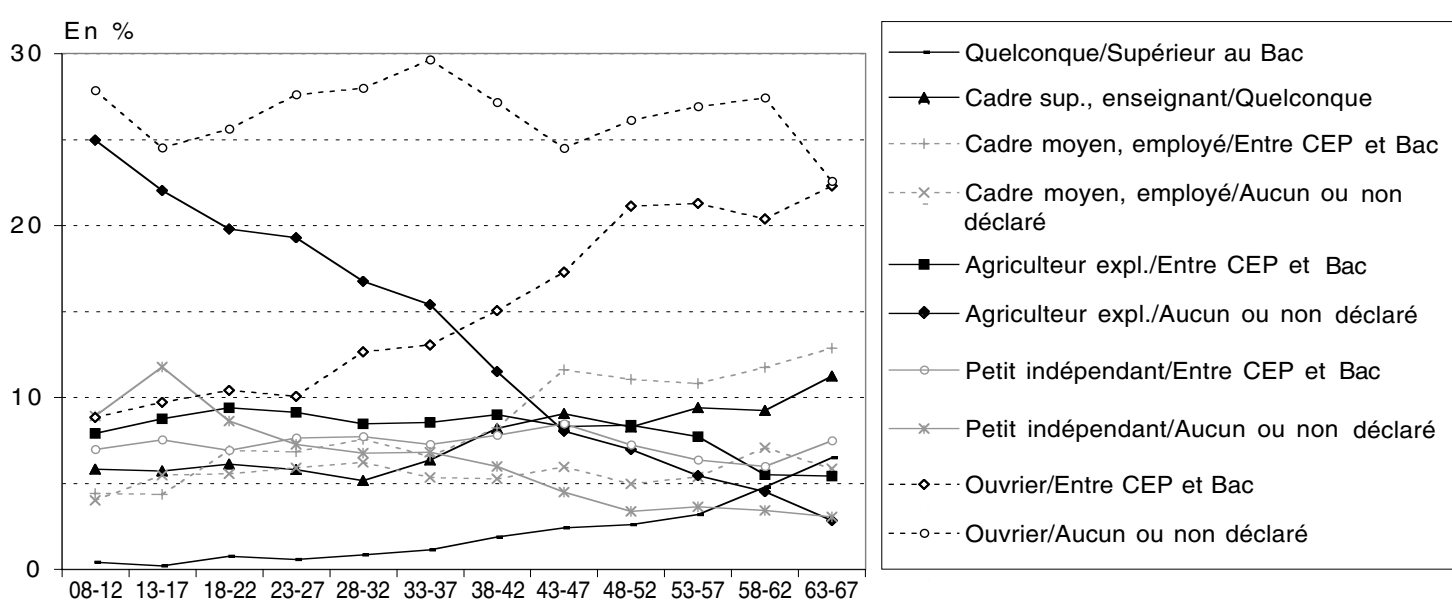

1. L'échantillon considéré ici a un effectif total de 66781 individus. Pour le champ et la source, on se reportera à l'encadré 1. Lecture : environ 25 \% des individus nés entre 1908 et 1912 avaient un père agriculteur exploitant et une mère sans diplôme (ou non déclaré). 
tous les cas, le mouvement le plus net est la très forte diminution, absolue ou relative, des familles où la mère n'avait aucun titre scolaire. À l'inverse, celles où la mère était très diplômée, c'est-à-dire titulaire d'un diplôme de l'enseignement supérieur, sont de plus en plus nombreuses à partir des générations nées durant la Seconde Guerre mondiale.

\section{Appréhender le diplôme} et sa dévalorisation éventuelle

Étudiant les inégalités sociales devant l'école, on se limitera à la formation initiale (les enquêtes le permettent le plus souvent, cf. encadré 1). On se contentera par ailleurs d'observer le diplôme le plus élevé que les personnes ont obtenu. Il s'agit ici d'un choix important. Certaines études privilégient au contraire, pour l'étude des inégalités devant le système scolaire, l'analyse de points de bifurcation fondamentaux de ce système: par exemple, l'entrée en $6^{\mathrm{e}}$, ou les différents paliers d'orientation - en fin de $5^{\mathrm{e}}$ ou en fin de collège - ou encore l'entrée dans l'enseignement supérieur. Cette perspective insiste donc sur la dynamique même des inégalités, telles qu'elles se construisent de bifurcation en bifurcation. Ici, à partir du diplôme le plus élevé obtenu, c'est le résultat final, en tant qu'il forme la synthèse de cette dynamique, et non ses étapes, qui est étudié. Le repérage du diplôme le plus élevé que les personnes ont obtenu est effectué, pour l'essentiel, au moyen de deux nomenclatures.

La première, en 7 postes, possède un degré de détail adapté à la fois aux générations anciennes (elle distingue entre aucun diplôme et titulaire du certificat d'études, ce qui est important, quoique souvent négligé, pour ces générations) et aux générations récentes (elle distingue entre bac +2 et bac +3 et plus, ce qui est essentiel pour ces dernières). Quel que soit son degré de finesse et de précision, cette nomenclature est formellement stable, c'està-dire qu'en l'utilisant on fait l'hypothèse implicite d'une stabilité, d'une «identité de valeur» des diplômes au cours du siècle. Hypothèse possible, peut-être même acceptable, mais forte.

Aussi la seconde nomenclature repose-t-elle sur la convention inverse, en supposant explicitement une dévalorisation des diplômes au cours du temps, d'une génération à l'autre. C'est la génération née en 1953 qui départage l'«avant» et l'«après », les équivalences suivantes étant alors supposées : le certificat d'études d'avant équivalant au brevet après, le brevet avant au baccalauréat après, le baccalauréat avant à bac +2 après, un diplôme d'études supérieures quelconque avant à un diplôme de niveau licence au moins après. Dans ce schéma, la valeur des diplômes professionnels (CAP et assimilé) est supposée inchangée.

Utiliser ces deux nomenclatures permet de mener deux analyses contrastées. La dévalorisation des diplômes qui est postulée par la seconde nomenclature est, à dessein, importante : elle représente, en termes d'années de scolarisation, de 2 à 3 ans. Les conclusions qui vaudront avec les deux nomenclatures auront donc une portée générale, puisqu'elles ne dépendront pas d'une évolution éventuelle de la valeur des diplômes.

\section{Des générations de plus en plus diplômées}

Sur le plan descriptif, les deux nomenclatures délivrent le même message, qui n'est d'ailleurs pas fait pour surprendre: des premières aux dernières générations, très forte diminution $\mathrm{du}$ poids des non-diplômés, et encore plus des titulaires du certificat d'études, diplôme qui a disparu en 1989, mais qui était moribond depuis vingt ans; hausse très importante des titulaires d'un CAP ou d'un BEP ; hausse des bacheliers, hausse des diplômés du supérieur, assez parallèle entre bac +2 et bac +3 et plus (cf. graphiques III et IV). Le « choc » provoqué par l'application, à partir de la génération née en 1953, de l'équivalence des diplômes résultant de leur dévalorisation supposée est assez accusé (cf. graphique IV), ce qui atteste du caractère sans doute excessif de la convention adoptée pour traduire cette dévalorisation. Du moins cet excès rend-il d'autant plus significative la confirmation des tendances du graphique III par celles du graphique IV - à l'exception, bien sûr, de la distorsion induite par la seconde nomenclature.

\section{L'allongement des études a été plus intense pour les filles que pour les garçons}

En dehors de la diffusion des études, se fait jour également une certaine «féminisation » : à l'inverse de ce qui avait cours autrefois, les filles font aujourd'hui des études plus longues

2. Ce renversement est commun à tous les pays développés. Dans tous, en effet, les filles réussissent aujourd'hui mieux à l'école que les garçons. Cette constatation est si générale qu'elle s'apparente à une «loi ». Pour un examen des causes possibles, se reporter à Baudelot et Establet (1992) ou à DuruBellat (1994 et 1995) 
Graphique III

Diplôme détaillé selon la génération (1)

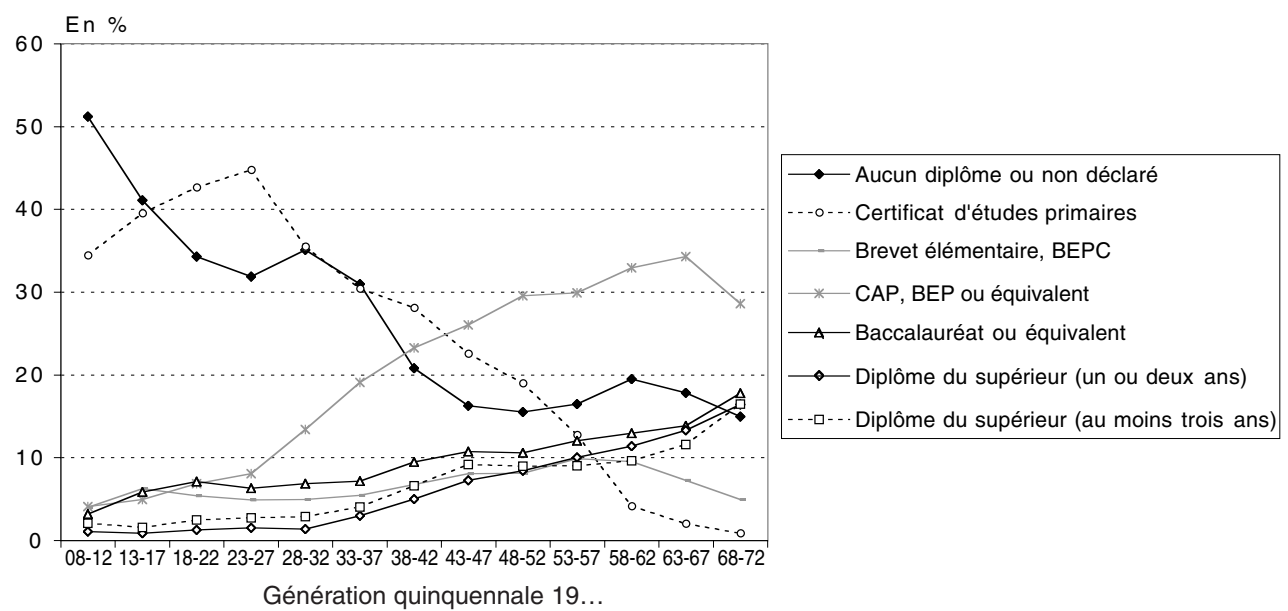

1. L'échantillon considéré ici a un effectif total de 240367 individus. Pour le champ et la source, on se reportera à l'encadré 1 Lecture : environ 51 \% des individus nés entre 1908 et 1912 n'ont obtenu aucun diplôme (ou non déclaré).

Graphique IV

Diplôme (en supposant une dévalorisation) selon la génération (1)

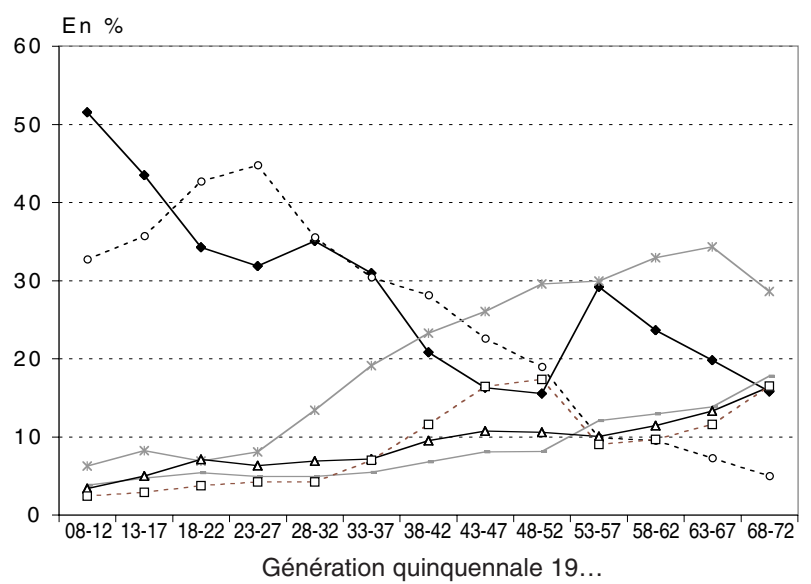

Naissance avant 1953 / à partir de 1953

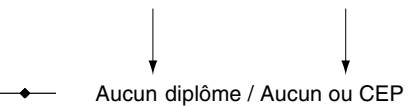

CEP / Brevet ou BEPC

CAP ou BEP / CAP ou BEP

Brevet ou BEPC / Baccalauréat

Baccalauréat / Supérieur (un ou deux ans)

Supérieur / Supérieur (au moins trois ans)

1. L'échantillon considéré ici a un effectif total de 244591 individus. Pour le champ et la source, on se reportera à l'encadré 1 . Par convention, la dévalorisation est concrétisée par un décalage entre les diplômes, suivant que l'individu est né avant ou à partir de 1953 (cf. encadré 1). Lecture : environ 51 \% des individus nés entre 1908 et 1912 n'ont obtenu aucun diplôme (ou non déclaré).

Graphique V

\section{Diplôme regroupé selon la génération et le sexe (1)}

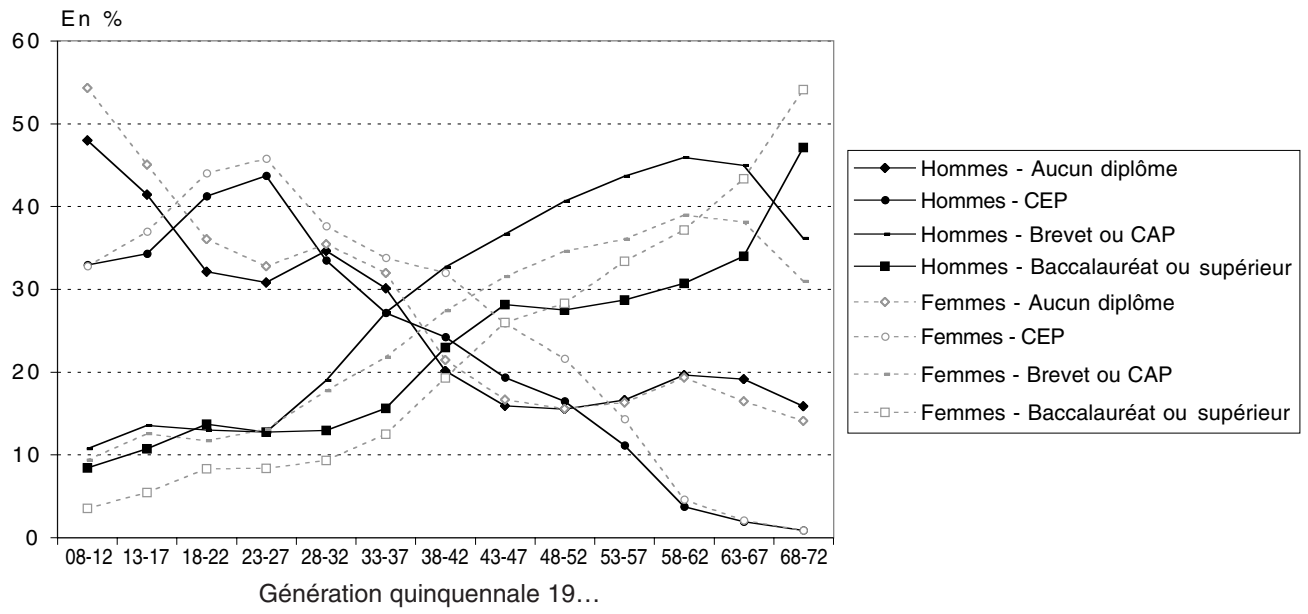

1. L'échantillon considéré ici a un effectif total de 244591 individus. Pour le champ et la source, on se reportera à l'encadré 1. Lecture : environ 54 \% des femmes nées entre 1908 et 1912 n'ont obtenu aucun diplôme (ou non déclaré). 
et plus réussies que les garçons (2). Le retournement a eu lieu au cours des années 60 . Il est nettement perceptible en termes d'années de scolarisation (Marchand et Thélot, 1997), mais aussi, comme ici, en termes de diplôme (cf. graphique V). La croissance de la part des filles titulaires du baccalauréat ou d'un diplôme du supérieur est beaucoup plus forte que celle relative aux garçons: de $3,5 \%$ à $54,1 \%$ des filles, contre de $8,4 \%$ à $47,1 \%$ des garçons. À l'inverse, sur la période, la diminution de la proportion de sans diplôme est plus prononcée pour les filles que pour les garçons. On peut identifier les générations dans lesquelles s'opère ce chassé-croisé à l'aide des durées nécessaires pour obtenir les différents diplômes. Il prend place à partir de la génération née en 1953. C'est aussi la première pour laquelle l'âge de fin de la scolarisation obligatoire a été fixé à 16 ans, et où la tendance à entrer en $6^{\text {e }}$ s'est accélérée. Au-delà de l'identification précise de cette génération, le retournement est spectaculaire : l'allongement des études, la massification et l'élévation des diplômes ont été plus prononcés pour les filles que pour les garçons. Cela conduit à se demander si les inégalités sociales devant l'école se sont davantage réduites pour elles que pour eux (cf. infra).

\section{Le poids de l'origine sociale}

La destinée scolaire est fortement liée à l'origine sociale, tant dans la première génération (personnes nées entre 1908 et 1912) que dans la dernière (personnes nées entre 1968 et 1972) (cf. tableau 1). Par exemple, dans un cas comme dans l'autre, ce sont les enfants d'enseignants, puis les enfants de cadres supérieurs (y compris gros indépendants) qui sont le plus souvent diplômés de l'enseignement supérieur ; à l'inverse, ce sont les enfants d'ouvriers non qualifiés (et agricoles) qui, le plus souvent, n'ont aucun diplôme. Mais l'allongement des études a été général et est perceptible dans tous les milieux sociaux, de sorte que l'accès au baccalauréat, ou aux études supérieures,

\section{Tableau 1 \\ Répartition des diplômes selon l'origine sociale pour les deux générations extrêmes (1908-1912 et 1968-1972)}

\begin{tabular}{|c|c|c|c|c|c|c|c|c|}
\hline Origine sociale & Génération & $\begin{array}{c}\text { Aucun } \\
\text { diplôme (1) }\end{array}$ & CEP & CAP, BEP & $\begin{array}{l}\text { Brevet, } \\
\text { BEPC (2) }\end{array}$ & Bac (3) & $\begin{array}{l}\text { Diplôme } \\
\text { supérieur } \\
\text { au bac }\end{array}$ & Ensemble \\
\hline Agriculteurs exploitants & $\begin{array}{l}1908-1912 \\
1968-1972\end{array}$ & $\begin{array}{r}66,1 \\
9,6\end{array}$ & $\begin{array}{l}28,4 \\
(0,8)\end{array}$ & $\begin{array}{r}2,3 \\
33,3\end{array}$ & $\begin{array}{l}1,3 \\
2,3\end{array}$ & $\begin{array}{r}1,1 \\
21,1\end{array}$ & $\begin{array}{r}0,9 \\
32,9\end{array}$ & $\begin{array}{l}100 \\
100\end{array}$ \\
\hline $\begin{array}{l}\text { Artisans, } \\
\text { petits commerçants }\end{array}$ & $\begin{array}{l}1908-1912 \\
1968-1972\end{array}$ & $\begin{array}{l}38,2 \\
12,8\end{array}$ & $\begin{array}{r}45,1 \\
1,4\end{array}$ & $\begin{array}{r}6,2 \\
31,4\end{array}$ & $\begin{array}{l}5,6 \\
5,6\end{array}$ & $\begin{array}{r}3,5 \\
15,8\end{array}$ & $\begin{array}{l}(1,4) \\
33,1\end{array}$ & $\begin{array}{l}100 \\
100\end{array}$ \\
\hline $\begin{array}{l}\text { Cadres supérieurs } \\
\text { (et gros indépendants) }\end{array}$ & $\begin{array}{l}1908-1912 \\
1968-1972\end{array}$ & $\begin{array}{r}19,7 \\
4,9\end{array}$ & $\begin{array}{l}24,9 \\
(0,1)\end{array}$ & $\begin{array}{r}12,5 \\
8,7\end{array}$ & $\begin{array}{r}12,3 \\
3,0\end{array}$ & $\begin{array}{l}16,0 \\
18,6\end{array}$ & $\begin{array}{l}14,6 \\
64,8\end{array}$ & $\begin{array}{l}100 \\
100\end{array}$ \\
\hline $\begin{array}{l}\text { Enseignants } \\
\text { (et assimilés) }\end{array}$ & $\begin{array}{l}1908-1912 \\
1968-1972\end{array}$ & $\begin{array}{r}(17,1) \\
4,2\end{array}$ & $\begin{array}{l}25,7 \\
(0,3)\end{array}$ & $\begin{array}{r}(7,3) \\
8,0\end{array}$ & $\begin{array}{r}(8,6) \\
2,5\end{array}$ & $\begin{array}{r}(21,6) \\
15,6\end{array}$ & $\begin{array}{r}(19,8) \\
69,4\end{array}$ & $\begin{array}{l}100 \\
100\end{array}$ \\
\hline Cadres moyens & $\begin{array}{l}1908-1912 \\
1968-1972\end{array}$ & $\begin{array}{r}15,2 \\
7,4\end{array}$ & $\begin{array}{l}35,1 \\
(0,3)\end{array}$ & $\begin{array}{l}16,5 \\
18,3\end{array}$ & $\begin{array}{r}15,6 \\
4,4\end{array}$ & $\begin{array}{l}12,4 \\
20,4\end{array}$ & $\begin{array}{l}(5,2) \\
49,3\end{array}$ & $\begin{array}{l}100 \\
100\end{array}$ \\
\hline Employés & $\begin{array}{l}1908-1912 \\
1968-1972\end{array}$ & $\begin{array}{l}39,1 \\
14,5\end{array}$ & $\begin{array}{r}38,1 \\
0,7\end{array}$ & $\begin{array}{l}10,3 \\
31,2\end{array}$ & $\begin{array}{l}5,5 \\
5,5\end{array}$ & $\begin{array}{r}4,1 \\
19,5\end{array}$ & $\begin{array}{l}(2,9) \\
28,6\end{array}$ & $\begin{array}{l}100 \\
100\end{array}$ \\
\hline $\begin{array}{l}\text { Contremaîtres } \\
\text { et ouvriers qualifiés }\end{array}$ & $\begin{array}{l}1908-1912 \\
1968-1972\end{array}$ & $\begin{array}{l}45,9 \\
19,1\end{array}$ & $\begin{array}{r}37,6 \\
0,8\end{array}$ & $\begin{array}{r}9,3 \\
35,2\end{array}$ & $\begin{array}{l}3,6 \\
5,5\end{array}$ & $\begin{array}{r}2,3 \\
18,1\end{array}$ & $\begin{array}{l}(1,3) \\
21,4\end{array}$ & $\begin{array}{l}100 \\
100\end{array}$ \\
\hline $\begin{array}{l}\text { Ouvriers non qualifiés } \\
\text { et agricoles }\end{array}$ & $\begin{array}{l}1908-1912 \\
1968-1972\end{array}$ & $\begin{array}{l}65,2 \\
27,3\end{array}$ & $\begin{array}{r}27,8 \\
1,7\end{array}$ & $\begin{array}{r}4,8 \\
38,2\end{array}$ & $\begin{array}{r}(1,1) \\
6,6\end{array}$ & $\begin{array}{l}(0,8) \\
14,1\end{array}$ & $\begin{array}{l}(0,3) \\
12,2\end{array}$ & $\begin{array}{l}100 \\
100\end{array}$ \\
\hline Ensemble & $\begin{array}{l}1908-1912 \\
1968-1972\end{array}$ & $\begin{array}{l}51,5 \\
15,0\end{array}$ & $\begin{array}{r}32,7 \\
0,8\end{array}$ & $\begin{array}{r}6,2 \\
28,6\end{array}$ & $\begin{array}{l}3,8 \\
5,0\end{array}$ & $\begin{array}{r}3,4 \\
17,7\end{array}$ & $\begin{array}{r}2,4 \\
32,9\end{array}$ & $\begin{array}{l}100 \\
100\end{array}$ \\
\hline \multicolumn{9}{|c|}{$\begin{array}{l}\text { 1. Ou diplôme non déclaré. } \\
\text { 2. Sans diplôme technique. } \\
\text { 3. Ou équivalent : BEI, BEC, BEH, etc. }\end{array}$} \\
\hline
\end{tabular}

Lecture : 66,1\% des enfants d'agriculteur exploitant nés entre 1908 et 1912 n'avaient aucun diplôme (ou non déclaré) ; c'est le cas de 9,6\% de ceux nés entre 1968 et 1972. Les pourcentages entre parenthèses reposent sur peu d'observations (moins de 10, " reconstituées ", cf. encadré 1) et sont donc assez fragiles.

Pour le champ et la source, on se reportera à l'encadré 1. 
qui était fermé aux enfants issus de familles populaires, s'ouvre sensiblement à ces milieux : parmi les plus jeunes, un tiers des enfants d'agriculteurs, un cinquième de ceux de contremaîtres et d'ouvriers qualifiés sont titulaires d'un diplôme de l'enseignement supérieur. Surtout, la façon dont les différents milieux sociaux ont profité de cet allongement général des études est variable. La comparaison des enfants d'agriculteurs et des enfants d'ouvriers non qualifiés et agricoles est particulièrement instructive. Alors que dans les générations anciennes les études étaient identiques dans ces deux milieux (66\% des enfants n'avaient pas de diplôme, $28 \%$ avaient le «certif »), il n'en est plus de même dans les générations récentes : les enfants d'agriculteurs font aujourd'hui, en moyenne, de meilleures études que les enfants d'ouvriers non qualifiés.
Avant d'analyser de façon approfondie l'évolution de ce lien entre milieu d'origine et études, il est utile d'en apprécier globalement la force au travers des différentes nomenclatures d'origine sociale et de diplôme retenues. On utilise pour cela un indice d'association global entre origine sociale et diplôme, dans les différentes générations (3) (cf. tableau 2). Les trois constats qui apparaissent alors sont les mêmes, que l'on suppose une stabilité ou une dévalorisation des diplômes.

3. Il s'agit $d u$ " V » de Cramer, compris entre $O$ (en cas d'absence complète de liaison entre origine sociale et diplôme) et 1 (en cas de liaison maximale). II s'exprime en fonction du Khi-deux de Pearson $\left(X^{2}\right)$, par la formule:

$$
\mathrm{V}=\left(\chi^{2} /[\mathrm{N} \cdot \min (\mathrm{NI}-1, \mathrm{NJ}-1)]\right)^{1 / 2}
$$

où $\mathrm{N}$ est le nombre d'observations, $\mathrm{NI}$ est le nombre de lignes du tableau et NJ le nombre de colonnes. Soulignons qu'il s'agit d'un indicateur global qui, en mesurant le lien entre origine sociale et diplôme, est aussi sensible aux distributions marginales du tableau et à leur variation (évolution de la structure sociale et allongement général des études).

Tableau 2

Force du lien global entre origine sociale et diplôme selon la génération (statistique du V de Cramer)

\begin{tabular}{|c|c|c|c|}
\hline & $\begin{array}{c}\text { Génération ancienne } \\
1908-1912\end{array}$ & $\begin{array}{c}\text { Génération médiane } \\
1938-1942\end{array}$ & $\begin{array}{c}\text { Génération récente } \\
1968-1972 \\
\text { (ou à défaut 1963-1967) }\end{array}$ \\
\hline \multicolumn{4}{|l|}{ Ensemble } \\
\hline \multicolumn{4}{|c|}{ En supposant stable la valeur des diplômes } \\
\hline Position du père & 0,238 & 0,205 & $0,193 \quad 0,185$ \\
\hline Position du père et diplôme de la mère & 0,257 & 0,239 & 0,232 \\
\hline Diplôme le plus élevé des deux parents & 0,280 & 0,263 & 0,255 \\
\hline \multicolumn{4}{|c|}{ En supposant une dévalorisation des diplômes } \\
\hline Position du père & 0,223 & 0,220 & $0,211 \quad 0,202$ \\
\hline Position du père et diplôme de la mère & 0,278 & 0,253 & 0,250 \\
\hline Diplôme le plus élevé des deux parents & 0,276 & 0,254 & 0,253 \\
\hline \multicolumn{4}{|l|}{ Hommes } \\
\hline \multicolumn{4}{|c|}{ En supposant stable la valeur des diplômes } \\
\hline Position du père & 0,281 & 0,212 & $0,198 \quad 0,193$ \\
\hline Position du père et diplôme de la mère & 0,309 & 0,254 & 0,234 \\
\hline Diplôme le plus élevé des deux parents & 0,334 & 0,270 & 0,263 \\
\hline \multicolumn{4}{|c|}{ En supposant une dévalorisation des diplômes } \\
\hline Position du père & 0,251 & 0,227 & $0,217 \quad 0,210$ \\
\hline Position du père et diplôme de la mère & 0,315 & 0,267 & 0,248 \\
\hline Diplôme le plus élevé des deux parents & 0,332 & 0,261 & 0,259 \\
\hline \multicolumn{4}{|l|}{ Femmes } \\
\hline \multicolumn{4}{|c|}{ En supposant stable la valeur des diplômes } \\
\hline Position du père & 0,240 & 0,205 & $0,191 \quad 0,182$ \\
\hline Position du père et diplôme de la mère & 0,274 & 0,236 & 0,252 \\
\hline Diplôme le plus élevé des deux parents & 0,291 & 0,261 & 0,270 \\
\hline \multicolumn{4}{|c|}{ En supposant une dévalorisation des diplômes } \\
\hline Position du père & 0,234 & 0,217 & $0,209 \quad 0,199$ \\
\hline Position du père et diplôme de la mère & 0,290 & 0,250 & 0,273 \\
\hline Diplôme le plus élevé des deux parents & 0,286 & 0,251 & 0,268 \\
\hline
\end{tabular}

Lecture : en supposant stable la valeur des diplômes, la force du lien global entre position du père et diplôme a diminué de 0,238 dans la génération 1908-1912 à 0,205 dans la génération 1938-1942, puis 0,185 dans la génération 1968-1972. La dernière colonne concerne la génération 1963-1967, puis 1968-1972 lorsque l'origine sociale est repérée par la position du père; elle concerne la seule génération 1963-1967 dans les autres cas.

Pour le champ et la source, on se reportera à l'encadré 1. 


\section{Le milieu culturel aurait davantage d'impact que le milieu social}

Constatation structurelle, d'abord. Le lien est systématiquement plus fort lorsque, pour caractériser le milieu d'origine des enfants, on introduit le diplôme de la mère en plus de la position du père. Incorporer les études maternelles apporte une information supplémentaire significative: la position du père ne résume pas, à elle seule, les handicaps ou les atouts sociaux devant l'école. D'ailleurs, si l'on accentue cette perspective en remplaçant la position sociale du père par son diplôme, c'està-dire en reliant le diplôme de l'enfant au diplôme le plus élevé de ses parents, l'association est presque toujours plus forte (14 cas sur 18). Ainsi, l'inégalité devant l'école s'exprime davantage comme une inégalité culturelle (via le diplôme de la mère ou celui des parents) que sous la forme d'une inégalité strictement sociale. Ce constat peut s'interpréter de deux façons. D'une manière plutôt taxinomique, c'est-à-dire en termes de classification, on conclura que l'introduction des études de la mère ou des parents, en plus ou à la place de la position du père, permet bien de préciser le milieu social d'origine, puisque, dans la société française, une dimension essentielle de la classe sociale est précisément la dimension scolaire ou culturelle. Ou bien, d'une manière plutôt réelle, on opposera les influences de l'héritage culturel et de l'héritage social sur la réussite scolaire, en attribuant alors au premier un rôle qui, non seulement n'est pas négligeable, mais excède même celui de l'héritage social. Et il est vrai, par exemple, que la réussite scolaire des enfants d'enseignants, même peu fortunés, est plus forte que celle des enfants d'indépendants, même fortunés.

\section{L'affaiblissement du lien global entre origine sociale et diplôme tient, pour une part, à l'allongement des études}

Constatation historique, ensuite. La force du lien global entre milieu d'origine et diplôme diminue des générations anciennes aux générations récentes. Pratiquement, quelles que soient les nomenclatures, donc les points de vue ( 38 fois sur 42 ), l'indice d'association décroît : les diplômes sont aujourd'hui moins dépendants du milieu d'origine et cette évolution a revêtu depuis 60 ans un caractère continu, en dépit d'une inflexion à la hausse, récente et passagère (cf. graphique VI). À la réflexion, cette moindre dépendance n'est pas étonnante dès lors que les études se sont généralisées au moins jusqu'à un certain niveau : quand l'entrée en $6^{e}$ devient la règle $(93 \%$ des enfants à partir de la génération née en 1960), alors qu'auparavant moins de la moitié des

\section{Graphique VI \\ Lien global entre origine sociale (position du père) et diplôme détaillé (sans hypothèse de dévalorisation) (1)}

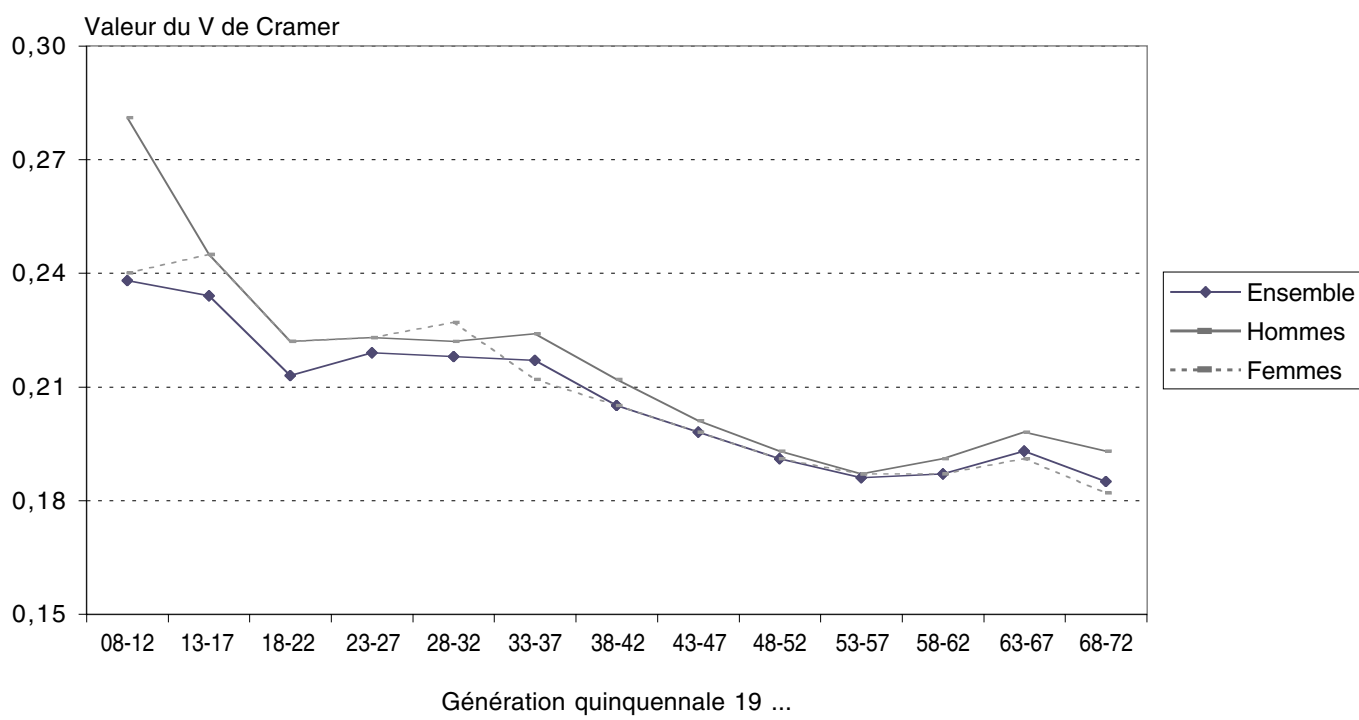

1. L'échantillon considéré ici a un effectif total de 240367 individus. Pour le champ et la source, on se reportera à l'encadré 1. Le lien est mesuré ici par le $\mathrm{V}$ de Cramer, indicateur global. Outre la force intrinsèque de ce lien, cet indicateur incorpore donc les effets de l'évolution de la structure sociale et de l'allongement général des études.

Lecture : appréciée sur l'ensemble des individus, la force du lien global entre position du père et diplôme diminue de 0,238 (génération 1908-1912) à 0,185 (génération 1968-1972). 
enfants y accédaient (par exemple, 41 \% dans la génération née en 1947), cet événement - l'entrée au collège - cesse d'être un palier ou une orientation spécifique, donc cesse d'être socialement discriminant. Ce raisonnement vaut pour l'ensemble des principaux points de bifurcation qui jalonnent la carrière scolaire. Il ne revêt certes pas la même force dès lors que l'on retient le diplôme final, c'està-dire le résultat de toute la carrière scolaire. Néanmoins, même atténué, cet effet purement mécanique de l'allongement des études explique, au moins en partie, l'affaiblissement du lien global entre origine sociale et diplôme. Encore conviendra-t-il de préciser plus loin quelle proportion de cet affaiblissement lui est strictement imputable.

\section{L'origine culturelle serait devenue plus déterminante pour les filles que pour les garçons}

Constatation à la fois structurelle et historique, enfin. Le lien global entre milieu d'origine et diplôme est, sur toute la période, presque toujours plus marqué pour les garçons que pour les filles (cf. graphique VI). Cependant, dans la génération la plus récente, il se révèle plus prononcé parmi celles-ci dès lors que l'on tient compte de l'héritage culturel, via les études de la mère ou le diplôme le plus élevé des deux parents (cf. tableau 2).

\section{Trois voies possibles pour préciser les rôles respectifs de la massification et de la démocratisation qualitative}

$\mathrm{Au}$ terme de cette approche descriptive, l'allongement général des études - la massification - et l'affaiblissement éventuel du lien entre origine sociale et diplôme - la démocratisation qualitative - constituent les ressorts majeurs de l'évolution des destinées scolaires en fonction de l'origine sociale, au fil des générations. Pour parvenir à une appréciation quantitative de leur importance respective et préciser ainsi l'évolution historique des inégalités sociales devant l'école, trois voies s'ouvrent au chercheur.

La première, assez ancienne, consiste à relier par une équation directe le plus haut niveau d'études atteint à différentes variables, dont l'origine sociale. L'analyse peut être individuelle ou par cohorte. Elle suppose d'avoir quantifié la mesure du niveau d'études sur laquelle ensuite un modèle de régression multiple est estimé. Les conclusions reposent alors sur l'évolution de génération en génération des coefficients reliant (toutes choses égales par ailleurs) plus haut niveau d'études atteint et variables décrivant le milieu d'origine. Intéressante et simple a priori, cette voie présente cependant un inconvénient: on a pu montrer que les coefficients de régression étaient affectés, non seulement par l'évolution $\mathrm{du}$ lien entre milieu d'origine et niveau d'études atteint, mais aussi par l'expansion générale de la scolarisation et la tendance à l'allongement des études (Mare, 1980, 1981).

Une seconde voie, beaucoup plus récente, consiste alors à utiliser des régressions logistiques visant à expliquer la probabilité, à un tournant de la carrière scolaire, de connaître telle suite ou telle autre : probabilité d'entrer en $6^{\mathrm{e}}$, d'entrer en seconde générale et technologique (en soi ou conditionnellement au fait d'être entré en $6^{\mathrm{e}}$ ), d'entrer dans le supérieur après le baccalauréat. Les variables explicatives incluent le milieu d'origine. Cette seconde voie est très féconde puisque, par cette analyse des principaux points de bifurcation, elle permet d'interpréter les inégalités : quand et comment elles se forment ou, au contraire, à quoi leur réduction est due. La généralisation de l'entrée en $6^{\mathrm{e}}$ est, par exemple, le principal facteur de réduction des inégalités selon Duru-Bellat et Kieffer (1999), l'entrée en seconde n'ayant pas ajouté à ce mouvement. Tout au contraire, la démocratisation induite par l'accès généralisé à la $6^{\circ}$ s'est accompagnée, selon ces auteurs, de carrières scolaires au collège plus inégalitaires dans les années 70 et 80 (4).

La troisième voie repose, non pas sur des analyses sur données individuelles, mais sur l'étude agrégée du lien entre milieu d'origine et diplôme. Elle consiste en une analyse de la succession, au fil des générations, des tableaux de contingence croisant origine sociale et diplôme, c'est-à-dire des tableaux d'effectifs, analogues à ceux qui forment la base du tableau 1 pour les deux générations extrêmes. C'est cette voie que nous emprunterons car, durant la dernière décennie, de nouveaux modèles statistiques ont apporté un progrès notable dans l'analyse de tels tableaux (Erikson et Goldthorpe, 1992; Xie, 1992 ; Goodman et Hout, 1998). Permettant de distinguer des changements d'ampleur limitée qui passaient auparavant inaperçus, ils rendent

4. Pour des analyses complémentaires du même type sur la France, se reporter à Brauns (1998). 
possible une analyse plus fine et se prêtent à une interprétation plus précise. En particulier, ces nouveaux modèles remettent en cause certaines conclusions antérieurement tirées en suivant la même voie (Goux et Maurin, 1995, 1997), conclusions qui doivent alors être réexaminées à la lumière de ces nouveaux outils.

Quelle que soit la méthode d'analyse, et audelà de leur spécificité, deux traits généraux se dégagent des travaux antérieurs.

\section{L'allongement des études fait l'unanimité des analyses préexistantes...}

En premier lieu, toutes les recherches enregistrent pour la France, mais aussi pour bon nombre des pays développés, l'allongement général des études, leur massification. Le phénomène est tellement ample, se voit si nettement «à l'œil nu », sans méthode d'analyse particulière, que ce premier aspect ne surprend pas. Par suite de l'allongement général des études, nombre d'enfants issus des couches moyennes ou des milieux populaires parviennent à des niveaux, obtiennent des diplômes qui, de fait, étaient presque entièrement inaccessibles à leur milieu d'origine : le baccalauréat, l'université sont ainsi, pour beaucoup de jeunes, des étapes ou des parcours qu'ils sont les premiers de leur famille, de leur parenté à connaître. Le niveau des connaissances qu'apportent les études n'ayant pas baissé (Joutard et Thélot, 1999), cette accession représente une réelle forme de démocratisation. Au sens propre: l'accession à certains niveaux ou stades est plus générale, moins socialement sélective, plus démocratique. La société et l'économie dans leur ensemble y trouvent d'ailleurs intérêt.

Ainsi, contrairement à ce qu'on lit parfois, l'allongement général des études est, en luimême, une forme de démocratisation, dès lors que ces dernières ne se transforment pas en « monnaie de singe », c'est-à-dire que le niveau des connaissances qu'elles apportent ne baisse pas, et qu'elles continuent à s'accompagner sur le marché du travail d'avantages substantiels - risque moins élevé d'être au chômage, salaires supérieurs -, ce qui est le cas.

\section{... alors que la réalité de la démocratisa- tion qualitative reste contestée}

Même s'il revêt des aspects bénéfiques, cet allongement général des études pourrait ne correspondre, on l'a dit, qu'à une simple translation vers le haut des niveaux de formation à la portée des jeunes originaires des différents milieux sociaux. Dans un tel cas, les écarts de destinée scolaire entre ces jeunes se trouveraient seulement déplacés vers des diplômes plus élevés, mais ils conserveraient une même intensité d'une génération à l'autre. Il y aurait alors massification sans démocratisation qualitative. Et c'est ici le second aspect qu'examinent les travaux qui se sont penchés sur l'évolution historique des inégalités sociales devant l'école. Ils se demandent si, au cours des dernières décennies et en plus de l'allongement général des études, une réduction qualitative des inégalités s'est fait jour, qui correspondrait à un affaiblissement du lien entre origine sociale et destinée scolaire, l'accès aux diplômes les plus élevés s'élargissant davantage pour les enfants d'origine sociale modeste qui en étaient les plus exclus auparavant. C'est là qu'apparaît une divergence dans les conclusions de ces travaux. Si tous s'accordent sur l'ampleur modeste de cette éventuelle démocratisation qualitative devant l'effet, spectaculaire lui, de l'allongement général des études, certains la tiennent pour réelle quoique limitée (par exemple Duru-Bellat et Kieffer, 1999), alors que d'autres la jugent inexistante ou trop faible pour être significative (par exemple Goux et Maurin, 1995, 1997).

\section{Quatre modèles pour séparer les facteurs de l'évolution des destinées scolaires au fil des générations}

Pour apprécier l'importance relative de l'allongement général des études et de la démocratisation qualitative, il est nécessaire d'isoler chacun des facteurs de l'interaction desquels résulte l'évolution des tables de destinée scolaire en fonction de l'origine sociale, au fil des générations. Ces facteurs sont au nombre de trois :

- l'évolution de la structure sociale qui fait que les membres des générations récentes ne proviennent pas des mêmes milieux sociaux que ceux des générations anciennes;

- l'allongement général des études ou massification qui fait que la répartition selon le diplôme s'est beaucoup transformée des générations anciennes aux générations récentes;

- le lien entre origine sociale et diplôme et sa variation éventuelle au fil des générations : dans chacune, ce lien est exprimé par une batterie de coefficients (les rapports des 
chances relatives) qui traduisent l'avantage (ou le handicap) dont disposent les enfants d'origine sociale $i$ par rapport à ceux d'origine sociale $i$ ' pour la possession du diplôme $j$ plutôt que celle du diplôme j' (cf. encadré 2).

À cet effet, des modèles statistiques emboîtés et de précision croissante seront ajustés à la série des tables de destinée scolaire selon l'origine sociale. Chaque modèle représente une étape dans la prise en compte progressive, et cumulée, des différents facteurs : le premier modèle n'intègre que le premier facteur (évolution de la structure sociale), le second conjugue les premier et deuxième facteurs (évolution de la structure sociale et allongement général des études), ces deux modèles ont en commun de supposer la stabilité temporelle du lien entre origine sociale et diplôme. Enfin, les troisième et quatrième modèles adjoignent aux deux premiers facteurs l'évolution du lien entre origine sociale et diplôme, le quatrième laissant à cette évolution davantage de degrés de liberté que le troisième. La qualité d'ajustement de chacun de ces modèles permet de conclure à l'importance relative plus ou moins grande des différents facteurs.

\section{Modèle 1}

La structure sociale ayant profondément évolué en France au cours des dernières décennies, l'origine sociale des membres des générations successives s'est beaucoup modifiée (cf. graphiques I et II), et le premier modèle consiste à postuler que cela constitue le seul facteur qui aurait évolué d'une génération à la suivante, la durée des études et la relation entre milieu d'origine et diplôme, elles, ne se transformant pas. Ce modèle revient donc à supposer que les variations observées dans les tableaux croisant origine sociale et diplôme ne seraient que le reflet de l'évolution de la structure sociale : parce que les enfants sont, dans les générations récentes, plus souvent issus de familles de cadres qu'autrefois, ils font des études plus longues. Bien entendu, ce modèle est très éloigné de la réalité. Il fait donc office de référence destinée à illustrer ce qui se serait passé en l'absence d'allongement des études et de modification du lien entre origine sociale et diplôme.

\section{Modèle 2}

Habituel dans ce type d'analyse, il postule que les tableaux croisant origine sociale et diplôme se sont transformés de génération en génération sous l'effet des deux facteurs struc- turels (évolution de la structure sociale, allongement général des études), la relation entre milieu d'origine et diplôme demeurant, elle, inchangée. Par rapport au modèle précédent, le modèle 2 incorpore donc la massification, mais il ne suppose aucune démocratisation qualitative, en raison de cette stabilité, d'une génération à l'autre, du lien entre milieu d'origine et diplôme. Ou, ce qui est équivalent, c'est ce deuxième modèle qui traduit l'idée que l'allongement général des études aurait laissé inchangés les chances et les handicaps respectifs des enfants des différents milieux face à l'école (5). Le modèle 2 , beaucoup plus riche et plus proche de la réalité que le précédent, s'ajuste assez bien aux observations, de sorte qu'en première approximation, on conclut souvent que la massification ne s'est pas accompagnée d'une démocratisation qualitative ou encore que la «massification sans réduction qualitative des inégalités » est le phénomène majeur de l'évolution.

\section{Modèles 3 et 4}

Majeur, mais peut-être pas unique, et c'est là qu'interviennent les deux autres modèles d'analyse, apparus récemment dans la littérature. Aux deux effets structurels déjà présents dans le modèle 2, ils adjoignent l'ultime facteur d'évolution, en autorisant le lien entre origine sociale et diplôme à varier d'une génération à l'autre au lieu d'être supposé constant. En plus de la massification que reflétait déjà le modèle 2 , les modèles 3 et 4 sont donc susceptibles de mettre en évidence une démocratisation qualitative s'ils s'ajustent nettement mieux aux données que le modèle 2 et si les paramètres supplémentaires estimés s'interprètent en ce sens. Le troisième modèle est très puissant pour identifier une tendance dominante dans l'évolution des inégalités sociales devant l'école. Il suppose que tous les rapports des chances relatives varient dans la même direction d'une génération à la suivante : soit qu'ils se rapprochent de 1 , ce qui correspondra à une démocratisation qualitative effective, soit qu'ils s'en éloignent, ce qui s'interprétera au contraire comme une inégalité croissante. Le quatrième modèle permet à ces rapports d'évoluer, non pas de façon uniforme, mais plus diversifiée, certains pouvant se rapprocher de 1 et les autres s'en éloigner. Plus souple que le

5. Il suppose en effet la constance temporelle des rapports des chances relatives : par rapport aux enfants de milieu d'origine i', les chances qu'ont les enfants de milieu d'origine i d'obtenir le diplôme j plutôt que le diplôme j' sont rigoureusement inchangées au fil des générations. 
Encadré 2

\section{LES MODÈLES STATISTIQUES}

Soit $g$ une génération quinquennale quelconque (parm $\mathrm{NG}$ ), $i$ et $i$ deux origines sociales quelconques (parmi $\mathrm{NI})$, $j$ et $j$ deux diplômes quelconques (parmi $\mathrm{NJ}$ ). On désigne par $m_{i j g}$ l'effectif d'individus d'origine sociale $i$ et titulaires du diplôme $j$ dans la génération $g$; il s'agit plus précisément d'un effectif estimé (maximum de vraisemblance) sous un modèle particulier. Le rapport des chances relatives ou odds ratio od $g$ mesure, dans la génération $g$, la force de l'association statistique entre les origines sociales $i$ et $i$ et les diplômes $j$ et $j$ :

$o d_{g}=\frac{m_{i j g} / m_{i j^{\prime} g}}{m_{i^{\prime} j g} / m_{i^{\prime} j^{\prime} g}}$ ou encore

$\log \left(\operatorname{od}_{g}\right)=\log \left(m_{i j g}\right)+\log \left(m_{i^{\prime} j^{\prime} g}\right)-\log \left(m_{i j^{\prime} g}\right)-\log \left(m_{i^{\prime} j g}\right)$ od $_{g}$ peut s'interpréter comme la mesure de l'avantage (ou du handicap) comparatif dont disposent, dans la génération $g$, les individus d'origine sociale $i$, par rapport à ceux d'origine sociale $i$, pour la possession du diplôme $j$ plutôt que celle du diplôme $j$. En particulier, $o d_{g}$ vaut 1 lorsque cet avantage (ou ce handicap) comparatif est absent, c'est-à-dire dans le cas remarquable d'indépendance statistique entre les origines sociales et les diplômes distingués. Le tableau de contingence croisant $\mathrm{NI}$ origines sociales avec $\mathrm{NJ}$ diplômes, c'est un ensemble de nombreux odds ratios (parmi lesquels $(\mathrm{NI}-1)(\mathrm{NJ}-1)$ sont indépendants) qui permet de décrire la force et la structure de l'association statistique entre les deux variables.

Une propriété importante du rapport des chances relatives est qu'il exprime l'association statistique indépendamment des distributions marginales, c'est-à-dire qu'il est insensible à une transformation de la distribution des origines sociales et/ou de celle des diplômes. Par conséquent, l'odds ratio permet d'apprécier la force " pure » ou « intrinsèque » du lien entre origine sociale et diplôme ainsi que son évolution éventuelle au fil des générations, en laissant de côté ce qui, dans la transformation des tableaux croisés, renvoie à l'allongement général des études ou au changement de la structure sociale. C'est précisément l'intérêt de la modélisation log-linéaire et log-multiplicative des tableaux de contingence que de permettre de formuler et d'éprouver des hypothèses relatives aux odds ratios (Bishop, Fienberg et Holland, 1975 ; Agresti, 1990 ; Powers et Xie, 2000). Depuis 25 ans, cette technique statistique a été beaucoup utilisée dans la recherche internationale sur la mobilité sociale entre générations (Goux et Maurin, 1997 ; Vallet, 1999). Ses développements récents sont ici appliqués à des tableaux de contingence à trois dimensions croisant origine sociale (O), diplôme (D) et génération $(\mathrm{G})$.

Modèle 1 : absence d'allongement général des études et constance des rapports des chances relatives

$\log \left(m_{i j g}\right)=\lambda+\lambda_{i}^{O}+\lambda_{i}^{D}+\lambda_{g}^{G}+\lambda_{i g}^{O G}+\lambda_{i j}^{O D}$

II est facile de vérifier qu'un tel modèle implique :

$\log \left(o d_{g}\right)=\lambda_{i j}^{O D}+\lambda_{i^{\prime} j^{\prime}}^{O D}-\lambda_{i j^{\prime}}^{O D}-\lambda_{i^{\prime} j}^{O D}$
Ainsi, le logarithme de chaque odds ratio s'exprime en fonction de paramètres qui ne sont pas indicés par $g$, ce qui correspond au fait que l'association statistique entre origine sociale et diplôme est supposée constante au cours des générations. La présence, au sein du modèle, des paramètres $\lambda$ (autres que ceux, $\lambda_{i j}$, relatifs à la liaison entre origine sociale et diplôme) garantit que le tableau de contingence estimé, croisant origine sociale, diplôme et génération, sera identique au tableau observé pour les marges correspondantes. En particulier, les paramètres $\lambda_{i g}^{O G}$ permettent de prendre en compte la transformation de la structure sociale intervenue au fil des générations.

En résumé, le modèle 1 postule que le tableau de contingence croisant origine sociale et diplôme ne s'est transformé, au cours du temps, qu'en raison de l'évolution de la structure sociale et que le lien intrinsèque entre les deux variables est resté inchangé. Il est estimé avec $\mathrm{NI}(\mathrm{NJ}-1)(\mathrm{NG}-1)$ degrés de liberté et peut encore être décrit comme le modèle d'indépendance statistique entre diplôme et génération, conditionnellement à l'origine sociale.

Modèle 2: constance des rapports des chances relatives

$\log \left(m_{i j g}\right)=\lambda+\lambda_{i}^{O}+\lambda_{j}^{D}+\lambda_{g}^{G}+\lambda_{i g}^{O G}+\lambda_{j g}^{D G}+\lambda_{i j}^{O D}$

De nouveau, un tel modèle implique :

$\log \left(o d_{g}\right)=\lambda_{i j}^{O D}+\lambda_{i^{\prime} j^{\prime}}^{O D}-\lambda_{i j^{\prime}}^{O D}-\lambda_{i^{\prime} j}^{O D}$

L'association statistique entre origine sociale et diplôme est donc supposée temporellement constante.Les paramètres supplémentaires $\lambda_{j g}^{D G}$ prennent désormais en compte l'allongement général des études, intervenu au fil des générations. Le modèle 2 postule donc que le tableau de contingence croisant origine sociale et diplôme ne s'est transformé, au cours du temps, qu'en raison de ce facteur et de l'évolution de la structure sociale, alors que le lien intrinsèque entre les deux variables est resté inchangé. Postulant l'absence d'interaction entre origine sociale, diplôme et génération, ce modèle a été désigné sous le terme de modèle de "démocratisation uniforme " par Goux et Maurin (1995, 1997). II est estimé avec (NI - 1)(NJ - 1)(NG - 1) degrés de liberté.

L'ajout, dans l'expression du modèle 2, des paramètres $\lambda_{i j g}^{O D G}$ conduirait au modèle saturé qui reproduirait parfaitement les données, mais ne les simplifierait en rien. Les (très nombreux) paramètres supplémentaires traduiraient alors la variation du lien entre origine sociale et diplôme, au fil des générations. Les modèles logmultiplicatifs qui suivent ont été introduits au cours de la dernière décennie dans la littérature spécialisée et ont l'intérêt de fournir des expressions parcimonieuses de cette variation.

Modèle 3 : évolution uniforme des rapports des chances relatives

$\log \left(m_{i j g}\right)=\lambda+\lambda_{i}^{O}+\lambda_{j}^{D}+\lambda_{g}^{G}+\lambda_{i g}^{O G}+\lambda_{j g}^{D G}+\beta_{g} \psi_{i j}$ 
Encadré 2 (fin)

On peut vérifier qu'un tel modèle implique :

$\log \left(\operatorname{od}_{g}\right)=\beta_{g}\left(\psi_{i j}+\psi_{i^{\prime} j^{\prime}}-\psi_{i j^{\prime}}-\psi_{i^{\prime} j^{\prime}}\right)$

Le logarithme de chaque odds ratio s'exprime comme le produit de deux ensembles : l'expression formée des paramètres $\psi$ traduit la forme générale - indépendante de la génération - de l'association statistique entre les origines sociales et diplômes mis en jeu ; le paramètre $\beta_{g}$ exprime, pour la génération considérée, la force relative de cette association. Le modèle 3 décompose donc le lien intrinsèque entre origine sociale et diplôme en une structure générale et un paramètre de niveau, caractéristique de chaque génération. II autorise ainsi une variation de tous les odds ratios dans la même direction - éloignement ou rapprochement de 1 -, celle-ci et son ampleur étant traduites par les paramètres $\beta_{g}$.

En réalité, seuls (NG - 1) paramètres de niveau peuvent être estimés car l'un doit être fixé à une valeur conventionnelle. Si l'on retient la valeur 1 pour la première génération, on peut déduire de l'expression précédente que l'estimation, pour une génération ultérieure, d'un paramètre inférieur à 1 correspondra à un affaiblissement de l'association statistique entre origine sociale et diplôme (rapprochement des odds ratios de 1); inversement, un paramètre supérieur à 1 s'interprétera comme un renforcement du lien (éloignement des odds ratios de 1).

Estimé avec (NI.NJ - NI - NJ)(NG - 1) degrés de liberté, ce modèle a été introduit par Erikson et Goldthorpe (1992) et par Xie (1992). Il a notamment été utilisé par Jonsson, Mills et Müller (1996) pour mettre en évidence, sur longue période, un affaiblissement du lien entre origine sociale et niveau d'études atteint en Allemagne et en Suède, alors qu'aucune tendance claire n'a pu être décelée en Grande-Bretagne. Le même modèle a aussi été employé par Vallet (1999) et a fait apparaître un lent accroissement de la fluidité sociale entre générations en France, de 1953 à 1993.

Exprimant, entre deux générations, la variation de l'association statistique entre origine sociale et diplôme par un unique paramètre, le modèle 3 s'avère très puissant pour déceler un changement. En effet, s une tendance dominante existe, dans les données observées, au sein des odds ratios, il sera le plus à même de la détecter. En contrepartie, le même modèle pourra être un peu fruste dans la description du changement intervenu car, dans la réalité, ce ne sont pas nécessairement tous les odds ratios qui se sont modifiés dans la même direction. Avantage et handicap proviennent du fait que, dans ce modèle, une même structure multiplicative capture à la fois l'association statistique entre origine sociale et diplôme et sa variation temporelle. Au prix d'une moindre parcimonie, il est possible de parvenir à une description plus fidèle en restreignant la structure multiplicative à la seule variation temporelle.

Modèle 4 : évolution diversifiée des rapports des chances relatives

$\log \left(m_{i j g}\right)=\lambda+\lambda_{i}^{O}+\lambda_{j}^{D}+\lambda_{g}^{G}+\lambda_{i g}^{O G}+\lambda_{j g}^{D G}+\lambda_{i j}^{O D}+\gamma_{g} \varphi_{i j}$

Un tel modèle implique :

$\log \left(o d_{g}\right)=\left(\lambda_{i j}^{O D}+\lambda_{i^{\prime} j^{\prime}}^{O D}-\lambda_{i j^{\prime}}^{O D}-\lambda_{i^{\prime} j}^{O D}\right)+\gamma_{g}\left(\varphi_{i j}+\varphi_{i^{\prime} j^{\prime}}-\varphi_{i j^{\prime}}-\varphi_{i^{\prime} j^{\prime}}\right)$ ce que l'on peut encore écrire : $\log \left(o d_{g}\right)=u_{i i^{\prime} j^{\prime}}+\gamma_{g} \nu_{i i^{\prime} j^{\prime}}$

L'association statistique entre origine sociale et diplôme est ici décomposée en une partie stable (terme $u_{i i^{\prime} j^{\prime}}$ ) et une partie variable selon la génération (terme $v_{i i^{\prime} j_{j}}$ ), mais l'importance relative de ces deux parts dépend du odds ratio considéré, c'est-à-dire des deux origines sociales et des deux diplômes mis en jeu. De même, la direction et l'ampleur de la variation de l'association statistique au fil des générations dépendent de l'odds ratio considéré. C'est en ce sens que ce modèle, estimé avec (NI.NJ - NI - NJ)(NG - 2) degrés de liberté et introduit par Goodman et Hout (1998), autorise une évolution diversifiée des rapports des chances relatives, certains se rapprochant de 1 , les autres s'en éloignant au fil des générations.

Une étude approfondie du modèle permet alors de préciser ce dernier aspect, selon les valeurs de $u_{i i^{\prime} j j}$, $v_{i i} i_{j}$, et $\gamma_{g}$. En fixant le paramètre de niveau à 1 pour la première génération, on démontre qu'une décroissance de $\gamma_{g}$ correspond à un rapprochement du odds ratio de 1 , comparativement à la première génération, dans les trois quarts du plan $(u, v)$ et à condition que $\gamma_{g}$ reste strictement supérieur à $-1-(2 u / v)$. À partir de cette caractérisation, il est alors possible d'évaluer, sur la base des valeurs $u_{i i^{\prime} j_{j}}$, $v_{i i^{\prime} j j^{\prime}}$ et $\gamma_{g}$ estimées, la proportion d'odds ratios qui, selon la génération, correspondent à un affaiblissement de l'association statistique entre origine sociale et diplôme. Ce sont ces proportions qui sont indiquées dans le corps du texte.

Les modèles utilisés dans cet article ont été estimés à l'aide du logiciel LEM développé par Jeroen K. Vermunt à l'Université de Tilburg (Pays-Bas). modèle 3 , le modèle 4 est alors susceptible de s'ajuster plus fidèlement aux données (cf. encadré 2).

Ces quatre modèles sont emboîtés: si le premier est vrai, le second l'est (mais la réciproque est fausse); si le second est vrai, le troisième l'est (mais la réciproque est fausse), etc. On peut donc facilement tester la qualité d'ajustement des uns par rapport aux autres.
Cela permet d'apprécier, en particulier, ce qu'apportent les troisième et quatrième modèles par rapport au deuxième.

Le jugement sur l'adéquation de ces modèles aux données est à la fois crucial pour l'interprétation qu'on en tirera, et délicat en raison de l'importance des échantillons sur lesquels nous nous fondons. Cette importance est évidemment une assurance de qualité pour 
les observations (6). Mais on sait que les tests d'adéquation des modèles aux données y sont sensibles : plus l'échantillon est grand, plus les tests usuels, par exemple celui du Khi-deux, conduisent à refuser tout modèle quel qu'il soit (sauf le vrai, le rigoureusement vrai, s'il existait...).

Ce qui est ici le cas. Aussi est-il habituel, pour contourner cette difficulté, de se fonder sur un indicateur qui tienne compte de cet effet taille, et, en même temps, de ce qu'on nomme la parcimonie du modèle, c'est-à-dire de sa capacité à être proche des données avec peu de paramètres. C'est évidemment une qualité appréciable, pour un modèle statistique, de reposer sur peu de paramètres, dès lors que les estimations auxquelles il conduit sont proches des données : on peut en effet, dans ce cas, reconstituer approximativement les observations à peu de frais, c'est-à-dire grâce à peu de paramètres (7). Certains indices permettent de tenir compte à la fois de l'adéquation du modèle (la statistique de test $G^{2}$ ), de sa parcimonie (liée à son degré de liberté $(d d l)$ : plus ce degré de liberté est petit, plus le modèle contient de paramètres) et de la taille de l'échantillon $(N)$. On utilise ici un tel indice, doté de bonnes propriétés et nommé bic (pour Bayesian Information Criterion) : bic $=G^{2}-(d d l) \ln (N)$.

\section{L'allongement général des études est le facteur central de l'évolution des destinées scolaires...}

Cette démarche trouve son aboutissement dans le tableau 3, où figurent les indices d'adéquation des quatre modèles, tels qu'ils ont été estimés sur les 13 tableaux de contingence croisant, pour les générations considérées, origine sociale (position du père) et diplôme. On a fait apparaître deux variantes selon la nomenclature des diplômes: soit que leur valeur est restée stable, soit qu'elle a diminué.

Aucun des modèles n'est admissible, bien que le quatrième le soit presque, même avec des échantillons aussi importants, ce qui est un indice de son excellente adéquation. Le deuxième modèle enregistre un progrès considérable par rapport au premier, vers les tables de destinée scolaire effectivement observées : pour un degré de liberté voisin (mais plus faible, bien sûr), la statistique de test est divisée par 10 et la proportion de personnes «mal classées » s'effondre. Cela confirme que l'allongement général des études est une explication centrale dans l'évolution des tables de destinée scolaire en fonction de l'origine sociale.

\section{...mais il s'est accompagné d'une démocratisation qualitative}

Est-elle suffisante ? C'est là qu'intervient la comparaison entre les modèles 2,3 et 4 . La procédure de tests emboîtés - légitime puisque les modèles le sont - conduit à refuser de s'en tenir au modèle 2. On considérera par exemple l'analyse générale (hommes et femmes), avec la nomenclature supposant stable la valeur des diplômes. Si l'on admet que le modèle 3 est juste, admettre, dans ce contexte, que le modèle 2 l'est, est refusé : la statistique de test 4 107,8 - 3 442,1 = 665,7 excède de beaucoup la borne habituelle, à 5 \% d'erreur, d'une variable du Khi-deux ayant $504-492=12$ degrés de liberté. De même, et encore plus, pour le modèle 4 : si 4 est tenu pour vrai, 2 ne peut être tenu pour vrai. On retrouve, bien entendu, l'effet de la taille, de sorte que ce raisonnement, juste en théorie, n'est pas absolument probant. Il serait encore juste si tous les effectifs étaient divisés par 10 - la statistique de test, divisée par 10, serait encore nettement supérieure à la borne -, ce qui laisse entendre que l'écart entre les modèles 2 et 3 et, encore plus, 2 et 4 , est considérable. D'ailleurs, la proportion de personnes mal classées au terme de la reconstitution des données diminue, en particulier du modèle 2 au modèle 4 .

Si l'on se réfère à l'indice bic, le modèle 4 paraît en effet nettement meilleur que le modèle 2 : la valeur de l'indice qui lui correspond est beaucoup plus faible, ce qui signifie que l'équilibre entre adéquation du modèle et multiplicité des paramètres est nettement meilleur. Non seulement le modèle 4 s'ajuste mieux que le modèle 2 , ce qui est évident puisqu'il l'englobe, mais il s'avère fécond de consentir à une cinquantaine de paramètres descriptifs supplémentaires (différence entre les degrés de liberté des modèles 2 et 4) pour réaliser ce progrès dans l'adéquation.

Le modèle 3 est également meilleur que le modèle 2, mais moins nettement. Il ne s'approche pas du modèle 4 , ni en qualité d'ajuste-

6. L'échantillon est de 240000 enquêtés environ, au total, c'està-dire toutes générations confondues. Sa taille varie entre 3300 personnes pour la génération 1913-1917 et 36100 pour la génération 1948-1952.

7. En effet, si l'objectif était de reconstituer les observations avec un modèle qui contienne autant de paramètres que l'on voulait, il suffirait de retenir le modèle saturé, celui qui a un paramètre par case du tableau, auquel cas le modèle serait parfait, mais inutilisable car dépourvu d'interprétation. 
ment - si on suppose que 4 est vrai, 3 ne peut être tenu pour vrai - , ni en équilibre entre cette qualité et sa parcimonie. Il a pour lui, cependant, de se prêter à une présentation simple, puisqu'il ne fait qu'ajouter 12 paramètres au modèle 2 , un paramètre par génération (en ayant fixé celui de l'une d'entre elles à une valeur quelconque). Le profil de ces paramètres au fil des générations est décroissant (cf. graphique VII), ce qui signifie que, dans le cadre de ce modèle, les rapports de chances ont évolué de façon uniforme vers un rapprochement de 1 , traduisant ainsi une tendance à l'affaiblissement du lien entre origine sociale et diplôme (cf. encadré 2). L'ampleur de cette baisse et le moment où elle est apparue seront discutés plus loin (8).

8. On retrouve ainsi une évolution décelée par Smith et Garnier (1986) sur l'enquête FQP de 1970. Utilisant un premier modèle log-multiplicatif proposé par Goodman en 1979, ils concluaient à " un déclin notable de la forte association entre profession du père et diplôme le plus élevé obtenu parmi les cohortes nées dans les années quarante ». Ils rectifiaient ainsi une conclusion antérieure liée à l'usage d'une méthode statistique insuffisamment puissante (Garnier et Raffalovich, 1984).

Tableau 3

\section{Indicateurs d'ajustement des quatre modèles}

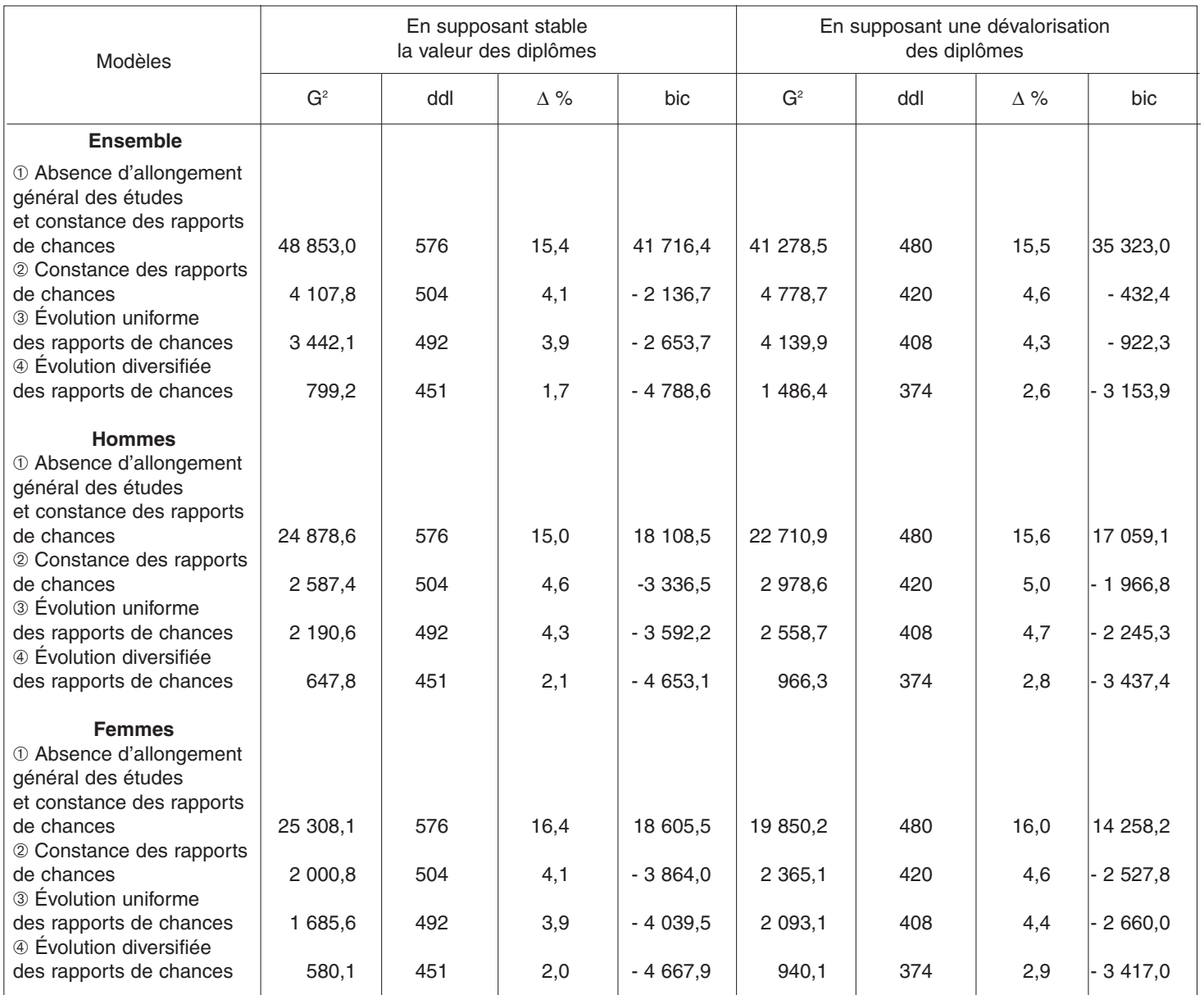

Lecture: les ajustements sont effectués à partir de 13 tables, chacune correspondant à une génération. Puisque les différentes générations ont été observées à plusieurs enquêtes, la table propre à une génération est l'addition des effectifs de chaque enquête pour celle-ci. Au minimum (générations extrêmes), il n'y a qu'une observation puisqu'elles n'ont été saisies qu'à une enquête. Au maximum, il y en a six. Les effectifs sont ceux des échantillons, mais en ayant pris soin de se fonder sur les résultats pondérés des enquêtes ramenés à l'effectif réellement enquêté (cf. encadré 1). Les effectifs sont les suivants : en supposant stable la valeur des diplômes, 240367 (ensemble), 127229 (hommes) et 113138 (femmes) ; en supposant une dévalorisation des diplômes, 244591 (ensemble), 129911 (hommes) et 114680 (femmes).

Cette taille très importante des échantillons explique qu'à strictement parler aucun modèle ne soit admissible. En effet, les bornes du $\chi^{2}$ sont, pour les degrés de liberté figurant dans la $2^{e}$ colonne, les suivantes : au seuil de $5 \%, 501,2$ pour 451 degrés de liberté et 419,8 pour 374 degrés de liberté par exemple. Les valeurs effectives du $\chi^{2}$ du rapport de vraisemblance $\mathrm{G}^{2}$ (1re colonne) sont beaucoup plus élevées, ce qui conduit à refuser ces modèles. Mais cela est purement imputable à la taille des échantillons. Si elle était divisée par 10 par exemple, pour être de l'ordre de 25000 - ce qui est une taille usuelle -, les modèles 3 et 4 seraient admissibles. D'où, pour contourner cette difficulté, le calcul d'un indicateur, bic, qui tient compte des trois paramètres $\mathrm{G}^{2}$, ddl et $\mathrm{N}$ : bic $=\mathrm{G}^{2}-(\mathrm{ddl})$ In $(\mathrm{N})$. Plus bic est faible, plus l'équilibre entre adéquation et parcimonie du modèle est bon. Raftery (1995) donne des ordres de grandeur pour choisir entre deux modèles : une différence de bic de 6 à 10 conduit à préférer fortement le modèle dont le bic est le plus faible; si elle excède 10, cette préférence doit être extrêmement forte. Avec une telle règle, il faut retenir les modèles 3 et 4 plutôt que le modèle 2 . $\Delta$ est la proportion de l'effectif total qui devrait changer de case pour que le tableau estimé soit identique au tableau observé : $\Delta$ est donc la demisomme des valeurs absolues des écarts, dans chaque case, entre proportions observées et proportions sous le modèle.

Pour le champ et la source, on se reportera à l'encadré 1. 
Ainsi, pour déterminant que soit l'allongement général des études dans l'évolution des destinées scolaires selon le milieu d'origine depuis le début du siècle, il n'est pas exclusif : en plus de la déformation de la structure sociale, en plus de l'allongement général des études (la massification), conclure à une certaine réduction des inégalités sociales devant l'école (la démocratisation qualitative) permet de mieux rendre compte de l'évolution observée. Il importe toutefois de ne pas perdre de vue que toute interprétation (concrétisée par le modèle retenu) n'est qu'approximative : se contenter du second modèle (massification sans réduction qualitative des inégalités), c'est se contenter d'une approximation plus grossière que retenir le troisième ou le quatrième. De surcroît, l'équilibre entre degré d'approximation et complexité du modèle paraît, au vu des observations, mieux satisfait avec ces deux derniers qu'avec le second.

\section{L'allongement des études explique $\mathbf{7 4 \%}$ de la différence des destinées scolaires entre les générations extrêmes, et la démocratisation qualitative, $14 \%$}

À titre d'exemple quantitatif, on a mesuré l'écart entre les tables de destinée scolaire en fonction de l'origine sociale, relatives aux deux générations extrêmes : celle des personnes nées entre 1908 et 1912 et celle des personnes nées entre 1968 et 1972. Après avoir éliminé l'effet de la différence de taille entre ces deux générations, on compare à cet effet le nombre de personnes ayant telle origine sociale et tel diplôme dans l'une et l'autre.

L'écart est considérable: si les deux générations avaient la même taille, il faudrait déplacer environ 2700000 personnes de la génération ancienne pour retrouver la distribution de la génération récente. Ce déplacement concernerait alors plus des deux tiers de la génération $(71,4 \%)(9)$. Il est possible de le décomposer en ses trois éléments constitutifs (cf. encadré 3) :

- effet de l'évolution de la structure sociale : $12 \%$ (300 000 personnes environ);

- effet de l'allongement général des études : $74 \%$ (2 000000 personnes environ);

- effet de la démocratisation qualitative : $14 \%$ (400 000 personnes environ).

9. Il est mesuré par la demi-somme des valeurs absolues des écarts...

$$
\frac{1}{2} \sum_{i, j}\left|m_{i j 1}-\left(\frac{n_{.1}}{n_{. .0}} m_{i j 0}\right)\right|
$$

où i représente l'origine sociale, j le diplôme, 0 la première génération, 1 la dernière, $\mathrm{m}$ les effectifs estimés sous le modèle 4 $\mathrm{n}$ les effectifs observés et le point indique la sommation sur l'indice correspondant. Plus précisément, on utilise ici, non pas les effectifs des échantillons, mais ceux-ci extrapolés à la population française.

Encadré 3

\section{DÉCOMPOSER L'ÉCART DE DESTINÉES SCOLAIRES ENTRE DEUX GÉNÉRATIONS} (1908-1912 et 1968-1972)

La façon de réaliser cette analyse du déplacement

$$
\frac{1}{2} \sum_{i, j}\left|m_{i j 1}-\left(\frac{n_{.1}}{n_{.0}} m_{i j 0}\right)\right|
$$

en ses trois composantes n'est pas unique et requiert des conventions car, comme on le sait, les valeurs absolues ne se décomposent pas aisément. D'où la démarche suivante.

On commence par estimer le modèle d'évolution diversifiée des rapports des chances relatives (modèle 4) sur les treize générations, ce qui fournit les effectifs $m_{i j 0}$ (génération 1908-1912) et $m_{i j 1}$ (génération 1968-1972). Quelle que soit la génération, ces tableaux ont les mêmes marges que les tableaux observés correspondants $\left(m_{i}=n_{i}\right.$ et $\left.m_{\cdot j}=n_{. j}\right)$. Par l'algorithme RAS, on peut ajuster aux effectifs $m_{i j 0}$ estimés pour la première génération les marges d'origine sociale et de diplôme de la dernière génération. Puisque cet algorithme a pour propriété remarquable de conserver les rapports des chances relatives du tableau initial, on obtient ainsi un tableau d'effectifs $I_{i j 1}$ qui représente ce qu'on aurait observé, pour la génération finale, si seuls l'évolution de la structure sociale et l'allongement général des études avaient joué. II est donc possible de calculer d'une part :

$$
\frac{1}{2} \sum_{i, j}\left|m_{i j 1}-l_{i j 1}\right|=415000
$$

qui s'interprète comme l'effet spécifique de la réduction qualitative des inégalités et d'autre part :

$$
\frac{1}{2} \sum_{i, j}\left|l_{i j 1}-\left(\frac{n_{.1}}{n_{. .0}} m_{i j 0}\right)\right|=2642000
$$

qui représente l'effet combiné des deux causes structurelles (évolution de la structure sociale et allongement général des études). D'où un premier résultat : la réduction qualitative des inégalités représente $14 \%$ du mouvement et les deux effets structurels combinés $86 \%$. 
Encadré 3 (fin)

II reste alors à séparer ces deux dernières composantes. Pour ce faire, on décompose l'évolution de la distribution marginale des diplômes entre les deux générations (car la réduction qualitative des inégalités n'affecte pas cette marge) en calculant d'abord :

$$
\alpha_{j 1}=\sum_{i} m_{i, 1}\left(\frac{m_{i j 0}}{m_{i .0}}\right)
$$

qui fournit la distribution par diplôme de la dernière génération dans l'hypothèse où seul l'effet de l'évolution de la structure sociale aurait joué. On peut alors calculer d'une part :

$$
\frac{1}{2} \sum_{j}\left|\alpha_{j 1}-\left(\frac{n_{.11}}{n_{. .0}} m_{. j 0}\right)\right|=379000
$$

qui représente précisément l'effet de cette évolution et d'autre part :

$$
\frac{1}{2} \sum_{j}\left|\alpha_{j 1}-m_{. j 1}\right|=2327000
$$

qui, symétriquement, mesure l'effet de l'allongement général des études. Ainsi, dans la combinaison des deux effets structurels, l'évolution de la structure sociale intervient pour $14 \%$ et l'allongement général des études pour $86 \%$.

On conclut, à l'aide de ces deux décompositions successives, que :

- l'effet de réduction qualitative des inégalités représente $14 \%$ du mouvement,

- l'effet de l'évolution de la structure sociale représente $14 \%$ x $86 \%$, soit $12 \%$,

- l'effet de l'allongement général des études représente $86 \% \times 86 \%$, soit $74 \%$,

et l'on peut enfin appliquer cette structure au déplacement global initial (2 700000 personnes).
Si l'on néglige la démocratisation qualitative, on rend donc compte de presque tout le mouvement : $86 \%$, soit $74 \%$ (allongement général des études) $+12 \%$ (évolution de la structure sociale).

On peut cependant appréhender l'importance de cette réduction qualitative des inégalités en comparant, pour les plus jeunes (génération 1968-1972), la table de destinée scolaire estimée sous le modèle 4 à celle que l'on aurait obtenue si seuls avaient joué l'évolution de la structure sociale et l'allongement général des études, le lien entre origine sociale et diplôme demeurant, lui, celui de la première génération (1908-1912). On évalue ainsi, pour les différentes origines sociales, la transformation des destinées scolaires dont est responsable la seule réduction qualitative des inégalités devant l'école. Ce sont environ 400000 personnes, soit $10 \%$ de la génération 1968-1972, qui ont accompli des études différentes de celles qu'elles auraient suivies en l'absence de tout affaiblissement du lien entre origine sociale et diplôme. Cette proportion diffère beaucoup selon le milieu d'origine : il s'agit de $50 \%$ des enfants d'agriculteurs, de $10 \%$ des enfants d'employés et d'ouvriers qualifiés, et de $7 \%$ à $9 \%$ de ceux de cadres, d'enseignants, d'artisans ou commerçants, et d'ouvriers non qualifiés. Tous les milieux sociaux sont donc concernés, mais en premier lieu la paysannerie : c'est surtout parmi les enfants d'agriculteurs que le niveau de diplôme atteint au terme de la scolarité s'est beaucoup élevé, en plus de ce qu'impliquait le seul allongement général des études (10). En l'absence de tout affaiblissement du lien entre origine sociale et destinée scolaire, dans la génération 1968-1972, environ 110000 enfants d'agriculteurs «supplémentaires » auraient dû quitter l'école sans diplôme ; en réalité, ils ont obtenu, soit un CAP ou un BEP (60 000), soit un baccalauréat (30 000), soit un BTS ou un DUT (20 000). L'impact de la démocratisation qualitative s'avère donc très significatif pour cette catégorie sociale. S'agissant d'un jeu à somme nulle, ces diplômes plus élevés des enfants d'agriculteurs exploitants - et, à un moindre degré, des enfants d'ouvriers - sont compensés par une moins bonne scolarité que ce qu'on aurait pu attendre, si le lien prévalant au début du siècle s'était perpétué, pour les enfants de cadres, d'enseignants, d'artisans ou commerçants, et d'employés (11). Mais cette compensation est plus diffuse et de moindre ampleur : comme on l'a déjà souligné, elle ne concerne, dans ces catégories sociales, qu'au plus $10 \%$ des enfants, contre la moitié des enfants d'agriculteurs.

10. Cependant, les enfants d'agriculteurs exploitants ne sont pas, à eux seuls, responsables de la tendance à l'affaiblissement du lien entre origine sociale et diplôme. En effet, lorsque les modèles 2 et 3 sont estimés en ignorant cette catégorie sociale, l'amélioration qu'apporte le modèle 3 reste fortement significative et le profil décroissant du graphique VII apparaît encore, l'ampleur de la variation n'étant que légèrement atténuée (le paramètre vaut 0,720 pour la génération 1968-1972 contre 0,651 dans le graphique VII).

11. Selon les estimations issues du modèle 4, la démocratisation qualitative intervenue depuis le début du siècle permet, dans la génération 1968-1972, à 100000 jeunes " supplémentaires » de milieux modestes (enfants d'agriculteurs exploitants, de contremaîtres et ouvriers qualifiés, ou d'ouvriers non qualifiés et agricoles) d'être titulaires du baccalauréat ou d'un diplôme de l'enseignement supérieur. Puisque la démocratisation qualitative, appréhendée comme une modification des rapports des chances relatives, n'affecte pas la distribution générale des diplômes, ce gain en diplômes plus élevés (ou cette diminution du handicap) doit être compensée par une perte équivalente (ou un amoindrissement de l'avantage) pour les autres catégories sociales. D'où l'expression "jeu à somme nulle ». 


\section{C'est dans l'après-guerre que la démocra-} tisation qualitative a joué à plein

Il est possible de préciser le profil temporel de cette réduction des inégalités. Quand on contraint la variation des rapports des chances relatives à être unique d'une génération à la suivante (modèle 3 ), le profil d'évolution est nettement orienté à la baisse. Le coefficient qui résume leur tendance passe de 1 (valeur conventionnelle pour la génération 19081912) et même 1,127 pour la génération suivante à 0,651 (génération 1968-1972), soit un taux moyen, obtenu par ajustement, de - 0,8 \% par an sur ces 60 ans. En réalité, la baisse n'est pas régulière (cf. graphique VII). Elle se produit surtout à partir de la génération née entre 1938 et 1942, donc pour les générations qui ont connu la scolarité d'aprèsguerre: durant les années 50 et 60 , l'école a fonctionné de façon moins inégalitaire. Un certain essoufflement de la démocratisation qualitative est ensuite enregistré pour les générations nées entre 1953 et 1967, comme si l'allongement de la scolarité jusqu'à 16 ans (effectif en 1969), l'entrée généralisée en $6^{\circ}$ (quasi effective en 1971) et, en fin de période, la création du collège unique, ne s'étaient pas traduits par un surcroît d'égalité des chances. Ce n'est que pour la dernière génération, née entre 1968 et 1972, qui a connu non seulement l'entrée généralisée en $6^{\mathrm{e}}$, mais aussi le mouvement vers le collège parcouru par tous, puis vers le lycée de masse, que la tendance à voir les inégalités décliner au-delà de l'allongement général des études reprend. Pour examiner si cette reprise de l'affaiblissement du lien entre origine sociale et destinée scolaire est durable, on ne peut se fonder sur des générations plus récentes, du moins si l'on retient une nomenclature détaillée des diplômes, puisqu'elles n'ont pas achevé leurs études. Mais le suivi de cohortes d'élèves par le ministère de l'Éducation nationale permet de constater que la tendance à la démocratisation qualitative des études, au moins secondaires, a effectivement repris : l'accès au baccalauréat est aujourd'hui nettement moins inégalitaire qu'il y a dix ans, et cela reste vrai si on se limite au baccalauréat général, et même au baccalauréat scientifique (cf. encadré 4).

$\mathrm{Au}$ lieu de contraindre les rapports des chances relatives à varier dans la même direction (modèle 3), on peut leur laisser une plus grande latitude d'évolution (modèle 4). L'ajustement de ce dernier modèle est nettement meilleur que celui du modèle 3, preuve que tous les rapports des chances relatives n'ont pas tendu à varier dans la même direction. Une analyse détaillée des contraintes qu'impose le modèle 4 et des estimations des rapports de chances auxquelles il aboutit montre que, selon la génération, 62 à $71 \%$ d'entre eux se sont rapprochés de 1 et traduisent ainsi un affaiblissement du lien entre origine

\section{Graphique VII \\ Lien entre origine sociale (position du père) et diplôme sous le modèle d'évolution uniforme des rapports de chances (1)}

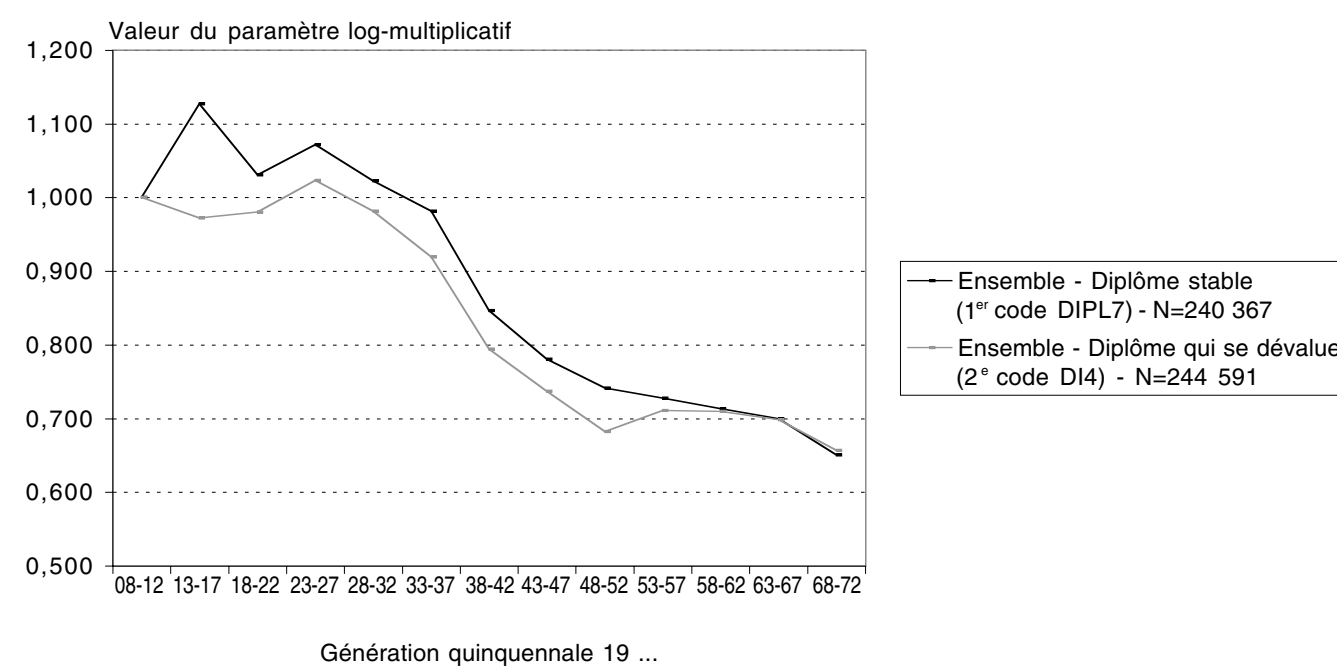

1. L'échantillon considéré ici a un effectif total de 240367 individus (en supposant stable la valeur des diplômes) et de 244591 individus (en supposant une dévalorisation des diplômes). Pour le champ et la source, on se reportera à l'encadré 1.

Lecture : en supposant stable la valeur des diplômes, le lien entre position du père et diplôme s'affaiblit de 1 (génération 1908-1912) à 0,651 (génération 1968-1972) 
Encadré 4

\section{L'ACCÈS AU BACCALAURÉAT DANS LES ANNÉES 90}

Le ministère de l'Éducation nationale suit régulièrement, année après année, des cohortes d'élèves au cours de l'enseignement secondaire : suivi des élèves entrés en $6^{\circ}$ en 1973 , puis suivi des élèves entrés en $6^{\circ}$ en 1980 , enfin suivi des élèves entrés en $6^{\text {e }}$ en 1989. En 1999 , cette dernière cohorte a presque complètement quitté l'enseignement secondaire, soit avant le baccalauréat, soit en l'ayant obtenu. Sans préjuger du cursus ultérieur dans l'enseignement supérieur, on peut donc étudier, selon le milieu d'origine, les chances d'accès au baccalauréat. Comme l'Ined avait fait la même chose pour les enfants sortis de CM2 en 1962, il est possible d'analyser, grâce à ces quatre cohortes, l'accès au baccalauréat au cours des années $60,70,80$ et 90 (cf. tableau).

Les inégalités sociales demeurent fortes à la fin des années 90, par exemple entre enfants d'ouvriers et enfants de cadres supérieurs ou de professeurs $52,5 \%$ des premiers, contre $85,3 \%$ des seconds, obtiennent leur baccalauréat, quel qu'il soit (on rappelle qu'il y a plus de 50 baccalauréats différents). En se limitant au baccalauréat général, le constat est identique : $22,5 \%$ des enfants d'ouvriers contre $71,0 \%$ des enfants de cadres supérieurs ou de professeurs l'atteignent. Ou encore, en envisageant le seul baccalauréat scientifique, $8,5 \%$ contre $38,6 \%$.

Mais ces inégalités sont moins fortes que pour les cohortes antérieures, dont le cursus secondaire s'est déroulé au cours des décennies précédentes. Par exemple, parmi les entrants en $6^{\circ}$ de 1980 , les chances d'obtenir plutôt que de ne pas obtenir le baccalauréat étaient 8,2 fois plus fortes pour les enfants de cadres supérieurs ou de professeurs que pour les enfants d'ouvriers ; le même rapport des chances relatives vaut 5,3 parmi les entrants en $6^{\circ}$ de 1989 . La réduction de l'écart est également visible avec le seul baccalauréat général en retenant les mêmes catégories sociales, 11,9 (cohorte 1980) et 8,4 (cohorte 1989). C'est encore le cas avec le seul baccalauréat scientifique : 11,5 (cohorte 1980) et 6,8 (cohorte 1989).

Pour une analyse plus générale de l'accès au baccalauréat prenant en compte les quatre cohortes et toutes les catégories sociales, il suffit d'appliquer aux données de ce tableau les modèles 2 et 3 . Auparavant, ces mêmes modèles seront estimés sur les 13 générations quinquennales étudiées. On utilise à cet effet l'indicateur principal de milieu d'origine (catégorie socioprofessionnelle du père en huit postes) et une nomenclature de diplômes dichotomique (n'a pas obtenu le baccalauréat, a au moins le baccalauréat). Avec le modèle 3 , la baisse des rapports des chances relatives est notable puisque la tendance diminue de 1 , valeur conventionnelle pour la génération 1908-1912 à 0,677 pour la génération 1968-1972, soit en moyenne - 0,6\% par an. On retrouve donc la conclusion du texte qui était fondée sur une nomenclature détaillée des diplômes. Le profil du graphique a aussi la même allure générale que le graphique VII. II montre que l'affaiblissement du lien a été sensible, pour les hommes comme pour les femmes, et légèrement plus rapide pour ces dernières.

La même méthode peut être appliquée aux quatre cohortes d'élèves: enfants sortis de CM2 en 1962, enfants entrés en $6^{e}$ en 1973, 1980 et 1989. Les sources et nomenclatures de milieu d'origine sont différentes, mais la tendance est également à la baisse, d'une cohorte à la suivante, et de l'ordre de $-1,1 \%$ par an en moyenne. La réduction des inégalités sociales d'accès au baccalauréat s'est donc prolongée jusqu'à aujourd'hui (fin des années 90). Elle s'est même accélérée entre les deux dernières cohortes : entrants en $6^{\circ}$ de 1980 et de 1989

En dehors de l'accès au baccalauréat, le cursus scolaire lui-même (par exemple l'ampleur des redoublements) se révèle moins inégalitaire au cours des années 90 que durant les années 80 (cf. par exemple Coëffic, 1998).

\section{Lien entre origine sociale (position du père) et possession/non-possession du baccalauréat sous le modèle d'évolution uniforme des rapports de chances (1)}

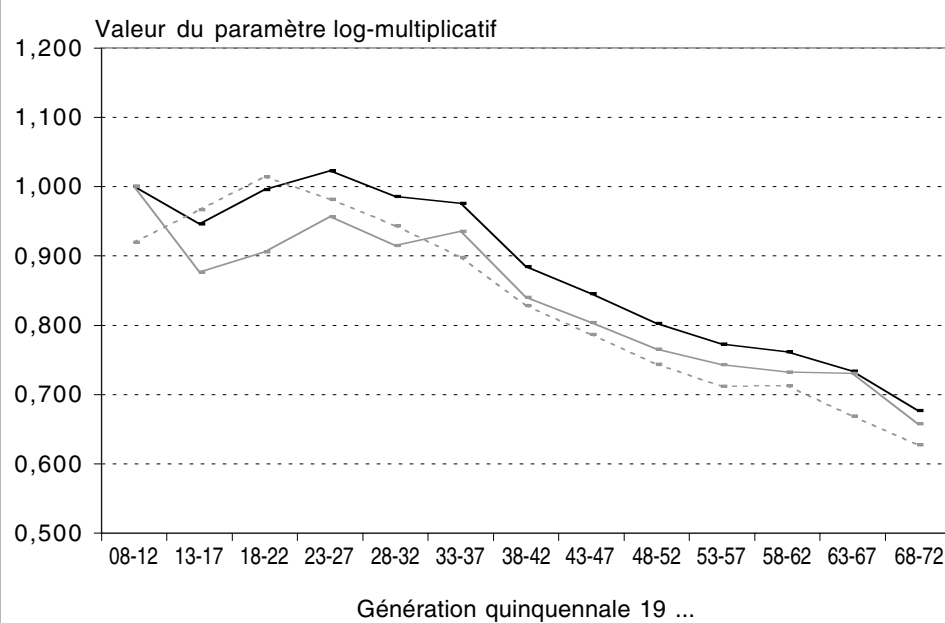

on se reportera à l'encadré 1 .

Lecture: apprécié sur l'ensemble des individus, le lien entre position du père et possession/non-possession du baccalauréat s'affaiblit de 1 (génération 1908-1912) à 0,677 (génération 1968-1972). 
Encadré 4 (fin)

Proportion d'élèves entrés en $6^{\circ}$ en $1962,1973,1980$ et 1989, qui ont obtenu le baccalauréat selon l'origine sociale

\begin{tabular}{|c|c|c|c|c|c|c|c|c|c|}
\hline \multirow{2}{*}{$\begin{array}{l}\text { Catégorie } \\
\text { socioprofessionnelle du père }\end{array}$} & \multicolumn{9}{|c|}{ Élèves entrés en $6^{\mathrm{e}}$ en : } \\
\hline & $1962(1)$ & 1973 & 1980 & $\begin{array}{l}\text { Dont bac } \\
\text { général }\end{array}$ & $\begin{array}{l}\text { Dont bac } \\
\text { C ou } D\end{array}$ & $\begin{array}{l}\text { Dont } \\
\text { bac C }\end{array}$ & 1989 & $\begin{array}{l}\text { Dont bac } \\
\text { général }\end{array}$ & $\begin{array}{l}\text { Dont } \\
\text { bac } S\end{array}$ \\
\hline Non-actifs et divers & 8,4 & 13,1 & 17,8 & 9,5 & 4,2 & 1,7 & 34,3 & 14,3 & 4,6 \\
\hline $\begin{array}{l}\text { Ouvriers, contremaîtres, } \\
\text { personnels de service }\end{array}$ & 11,3 & 16,8 & 25,9 & 12,6 & 4,5 & 1,5 & 52,5 & 22,5 & 8,5 \\
\hline Agriculteurs & 14,8 & 24,4 & 38,9 & 17,2 & 9,0 & 3,2 & 71,1 & 39,5 & 18,7 \\
\hline Artisans, commerçants & 23,4 & 30,1 & 39,0 & 24,5 & 9,2 & 2,9 & 58,7 & 31,0 & 12,0 \\
\hline Employés & 24,8 & 29,9 & 38,7 & 22,7 & 8,6 & 3,6 & 60,0 & 32,4 & 12,0 \\
\hline Cadres moyens, instituteurs & 49,3 & 48,8 & 57,3 & 39,7 & 18,1 & 7,6 & 76,3 & 51,7 & 23,0 \\
\hline $\begin{array}{l}\text { Cadres supérieurs, } \\
\text { professions libérales, } \\
\text { chefs d'entreprise, professeurs }\end{array}$ & 54,6 & 66,3 & 74,1 & 63,1 & 35,1 & 18,6 & 85,3 & 71,0 & 38,6 \\
\hline $\begin{array}{l}\text { Ensemble } \\
\text { (moyenne de la génération) }\end{array}$ & 20,6 & 28,1 & 38,5 & 24,3 & 10,9 & 4,8 & 62,7 & 37,0 & 16,6 \\
\hline
\end{tabular}

1. Les proportions de 1962 concernent les élèves sortis de CM2 et non les seuls élèves entrés en $6^{e}$. Elles ont été légèrement corrigées à partir des données initiales publiées par l'Ined pour couvrir environ 95 \% d'une génération.

Lecture : parmi les enfants d'ouvriers, contremaîtres et personnels de service, 16,8\% des entrants en $6^{\circ}$ de 1973 et $52,5 \%$ des entrants en $6^{e}$ de 1989 ont obtenu le baccalauréat (quel que soit le nombre d'années nécessaires à cette obtention).

Source : panel national d'élèves sortant de l'école primaire (recrutement 1962), INED; panels nationaux d'élèves du second degré (recrutements 1973, 1980 et 1989), ministère de l'Éducation nationale.

sociale et diplôme. Les tendances à une réduction qualitative des inégalités devant l'école ne se manifestent donc pas de façon uniforme, mais elles sont majoritaires.

Il est difficile d'aller beaucoup plus loin dans le cadre de cet article. En distinguant 8 origines sociales et 7 niveaux de diplôme, on dénombre en effet 588 rapports des chances relatives différents. À partir des estimations du modèle 4, il convient néanmoins de souligner que, parmi les 21 rapports de chances qui permettent d'opposer les cadres supérieurs et gros indépendants aux agriculteurs exploitants, seuls 5 ne correspondent, pour aucune génération, à un affaiblissement du lien entre origine sociale et diplôme ; c'est le cas de 7 sur 21 en remplaçant les agriculteurs par les contremaîtres et ouvriers qualifiés, de 10 sur 21 en leur substituant les ouvriers non qualifiés et agricoles. Dans ces trois contrastes entre catégories sociales, les cas qui ne correspondent pas à un affaiblissement du lien sont donc minoritaires et le résultat est identique en remplaçant les cadres supérieurs et gros indépendants par les enseignants (et assimilés). On confirme ici, par ailleurs, que la démocratisation qualitative a davantage concerné les enfants d'agriculteurs et d'ouvriers qualifiés que ceux d'ouvriers non qualifiés.

\section{L'origine sociale des filles, autrefois plus discriminante que celle des garçons, exerce aujourd'hui une influence comparable}

Toute l'analyse précédente peut être conduite par sexe. On s'aperçoit alors, en utilisant le troisième modèle, qu'au début du siècle et jusqu'aux générations nées avant la Seconde Guerre mondiale, le lien entre origine sociale et diplôme était nettement plus fort pour les filles que pour les garçons (cf. graphique VIII). La conclusion est donc opposée à celle qui se dégageait des inégalités brutes (cf. tableau 2 et graphique VI). Ầ nouveau, c'est à partir de la génération 1938-1942, celle qui a suivi une scolarité après-guerre, que l'évolution se fait jour: le lien entre origine sociale et diplôme a désormais, chez les garçons et les filles, une force voisine, et même presque identique pour les deux générations les plus récentes (personnes nées entre 1963 et 1972). Ainsi, la tendance à la réduction qualitative des inégalités sociales a été plus prononcée parmi les filles que parmi les garçons : - 0,9\% contre - 0,7 \% par an, en moyenne. Aujourd'hui, l'inégalité sociale devant l'école, une fois écartés la déformation de la structure sociale et l'allongement général des études, est analogue pour les garçons et les filles, peut-être même très légèrement inférieure parmi celles-ci. 
Cette conclusion provient d'une analyse séparée des inégalités devant l'école, c'est-àdire entre garçons de milieux sociaux différents d'une part, entre filles de milieux sociaux différents d'autre part. Il faut compléter l'examen en mêlant garçons et filles car il est possible que, dans les générations anciennes, et, encore plus, récemment, ce soit dans la dimension croisée du milieu d'origine et du sexe que résident les inégalités les plus frappantes: la réussite scolaire oppose peutêtre aujourd'hui, et de plus en plus, les filles de cadres et d'enseignants aux garçons des familles ouvrières. D'où l'intérêt d'une analyse croisée, et non plus séparée, de l'évolution des inégalités, là encore de génération en génération.

Le lien global, mesuré par le même indicateur que dans le tableau 2, a d'abord diminué, puis s'est stabilisé entre les générations 1948 et 1967. Enfin, tout récemment, il diminue de nouveau (génération 1968-1972). Si l'on isole la tendance d'évolution des rapports des chances relatives, comme dans l'analyse séparée des garçons et des filles, elle apparaît à la baisse et d'ampleur analogue: ils ont décru, de la première à la dernière génération, au rythme de - 0,8 \% par an en moyenne, leur diminution (rapprochement de 1) apparaissant également à partir de la génération née entre 1938 et 1942. Ainsi, contrairement à ce que l'on aurait pu penser, la réduction qualitative des inégalités est apparente, non seulement dans l'analyse séparée des garçons et des filles, mais aussi dans l'analyse croisée qui les mêle. La thèse selon laquelle de nouvelles inégalités se seraient fait jour entre les filles de certains milieux sociaux et les garçons d'autres milieux ne paraît pas confirmée dans cette approche d'ensemble.

\section{La démocratisation qualitative : une conclusion indépendante des enquêtes...}

Il reste à examiner l'impact du cadre statistique sur les conclusions portées jusqu'ici : ces résultats sont-ils indépendants des enquêtes et de la manière de les utiliser, ou encore des nomenclatures employées (on s'est contenté jusqu'à présent de la seule position sociale du père et on a supposé que les diplômes avaient conservé leur valeur au cours du temps)?

En ce qui concerne la dépendance aux enquêtes, au lieu d'estimer les modèles sur le cumul de toutes, on peut les estimer séparément sur chacune. Il y a 7 enquêtes, à partir desquelles peuvent donc être établis 7 profils d'évolution du paramètre qui mesure la force $\mathrm{du}$ lien entre position sociale du père et diplôme dans l'hypothèse d'une variation uniforme des rapports des chances relatives (modèle 3) (cf. graphique IX) (12). Ces profils

12. Le graphique IX présente les résultats d'estimations séparées, effectuées enquête par enquête, puis calées au point d'origine sur la valeur caractéristique de l'enquête FQP 1977, alors que le graphique de l'encadré 1 est établi à partir d'une estimation unique, fondée sur l'ensemble des tableaux initiaux génération $x$ enquête, au nombre d'une cinquantaine.

\section{Graphique VIII \\ Lien entre origine sociale (position du père) et diplôme (code détaillé) sous le modèle d'évolution uniforme des rapports de chances (1)}

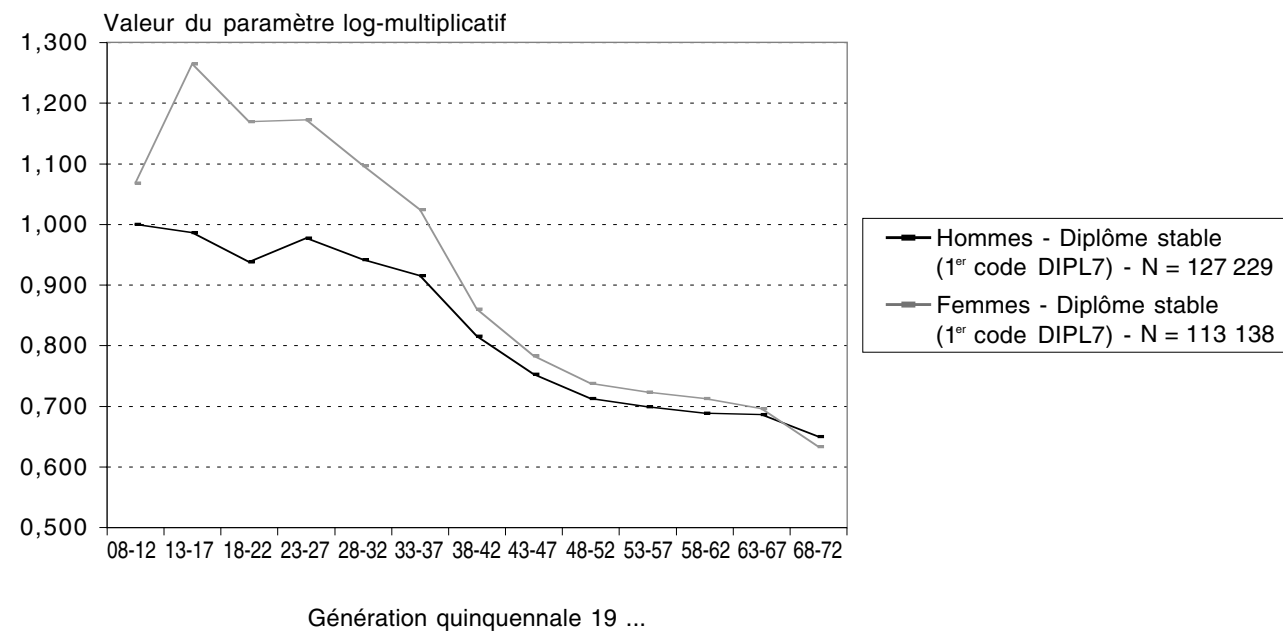

1. L'échantillon considéré ici a un effectif total de 127229 hommes et de 113138 femmes. Pour le champ et la source, on se reportera à l'encadré 1. Le modèle a été estimé simultanément sur les tableaux relatifs aux hommes et aux femmes.

Lecture : en supposant stable la valeur des diplômes, le lien entre position du père et diplôme s'affaiblit de 1 (génération 1908-1912) à 0,650 (génération 1968-1972) pour les hommes et de 1,068 à 0,633 pour les femmes. 
Graphique IX

Lien entre origine sociale (position du père) et diplôme (code détaillé) sous le modèle d'évolution uniforme des rapports de chances (1)

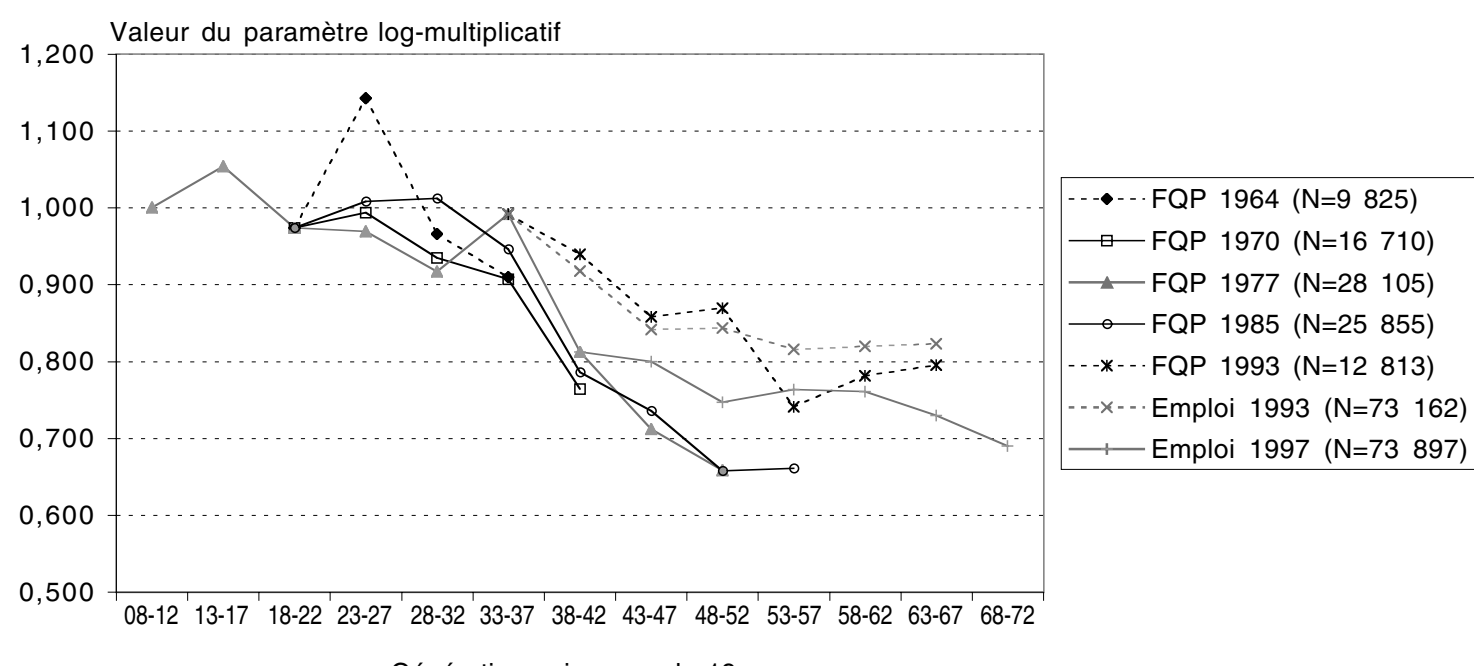

Génération quinquennale 19 ...

1. Pour le champ et la source, on se reportera à l'encadré 1. Le modèle a été estimé séparément sur chacune des 7 enquêtes. Pour les besoins de la comparaison et par convention, la valeur obtenue pour l'enquête FQP 1977 a ensuite été retenue comme point d'origine pour les autres enquêtes.

Lecture : en supposant stable la valeur des diplômes et selon les données de l'enquête FQP 1985, le lien entre position du père et diplôme s'affaiblit de 0,974 (génération 1918-1922) à 0,661 (génération 1953-1957).

confirment la tendance du paramètre à la baisse. Cependant, l'ampleur de celle-ci est variable d'une enquête à l'autre : certaines n'indiquent même pas de reprise récente de la diminution (en particulier l'enquête FQP 1993). Ainsi, pour chaque génération, la valeur du paramètre peut varier selon l'enquête. L'ampleur de ces fluctuations culmine à $32 \%$ (de 0,657 à 0,869 pour la génération 1948-1952, entre les enquêtes FQP 1985 et FQP 1993). Elle se situe dans la plupart des cas entre $8 \%$ et $20 \%$, et la moyenne, $17 \%$, donne un ordre de grandeur de la précision du coefficient. L'amplitude de ces fluctuations est donc sensiblement inférieure à la diminution, en 60 ans, du paramètre qui mesure la force du lien entre position du père et diplôme (35 \%). La réalité de la baisse est donc solidement établie, même si son ampleur demeure un peu imprécise. Au total, alors qu'il pourrait sembler imprudent de ne s'appuyer que sur une seule enquête, se fonder sur toutes à la fois confère, au contraire, une certaine force au constat (cf. encadré 1).

\section{... des nomenclatures rendant compte du diplôme...}

La tendance à la réduction qualitative des inégalités se révèle également indépendante de la nomenclature de diplômes utilisée, même si cette dernière affecte, en partie, le rythme de la décroissance mise au jour.
L'hypothèse d'une dévalorisation des diplômes au cours du temps conduit à des ajustements moins précis des modèles (cf. les colonnes correspondantes du tableau 3 ). Toutefois, elle ne modifie pas le sens général des conclusions : le troisième modèle et, surtout, le quatrième s'ajustent beaucoup mieux que le deuxième. L'utilisation de cette nomenclature confirme donc bien la réduction de l'intensité du lien entre origine sociale et diplôme. En supposant que les rapports des chances relatives ont varié uniformément d'une génération à la suivante (modèle 3), l'évolution du paramètre mesurant la force de ce lien est voisine de celle qui apparaissait en considérant stable la valeur des diplômes (cf. graphique VII). Seul le rythme de la décroissance est un peu atténué : - 0,7 \% par an, en moyenne, contre - 0,8\% précédemment.

On a utilisé de même quatre nomenclatures binaires qui se bornent à enregistrer la possession ou l'absence de tel ou tel diplôme : avoir (au moins) le certificat d'études primaires ou non, avoir (au moins) le brevet, le CAP ou le BEP ou non, avoir (au moins) le baccalauréat ou non, avoir un diplôme de l'enseignement supérieur ou non. Ce faisant, on conclut de nouveau à un affaiblissement du lien entre origine sociale et diplôme, mais, avec le modèle 3 , le rythme moyen de décroissance varie selon la nomenclature dichotomique utilisée : respectivement $-0,5 \%,-0,8 \%$, 
- 0,6 \% et - 0,8\% par an (les résultats qui concernent la troisième dichotomie, celle relative au baccalauréat, figurent dans l'encadré 4).

La réduction qualitative des inégalités devant l'école se révèle donc indépendante de la nomenclature de diplômes employée, le choix de cette dernière ne jouant que sur le rythme moyen à attribuer à cette diminution: entre $-0,5 \%$ et $-0,8 \%$ par an.

\section{... et de la nomenclature adoptée pour l'origine sociale}

L'affaiblissement du lien entre origine sociale et diplôme se révèle, enfin, indépendant de la manière d'appréhender le milieu d'origine. L'introduction du diplôme le plus élevé des deux parents permet en outre de préciser que les inégalités culturelles devant l'école se sont moins sensiblement réduites que les inégalités sociales.

On a estimé en effet les mêmes modèles en utilisant cinq autres définitions du milieu d'origine que la position du père : position de la mère, position croisée des deux parents, position et diplôme du père, position du père et diplôme de la mère, enfin diplôme le plus élevé des deux parents. L'usage de ces cinq nomenclatures confirme la tendance à la réduction qualitative des inégalités devant l'école: le paramètre habituel du modèle 3 diminue dans chaque cas, tant pour l'ensemble que pour les hommes et les femmes considérés séparément. Cependant, l'ampleur de cette décroissance varie selon la façon d'appréhender le milieu d'origine. La fourchette est analogue à celle obtenue en modifiant la nomenclature des diplômes: de $-0,5 \%$ à $-0,8 \%$ en moyenne par an pour l'ensemble, de - 0,5\% à - 0,8 \% pour les hommes et de - 0,6 \% à $-0,9 \%$ pour les femmes, ce qui constitue ainsi des écarts sensibles. De nouveau, la réduction apparaît plus marquée parmi les femmes : tel est le cas avec quatre des cinq nomenclatures. On confirme en outre que, jusqu'aux générations nées à la fin des années 30, le milieu d'origine était, du point de vue des études, plus discriminant pour les filles que pour les garçons alors que, dans les générations récentes, son influence est devenue comparable. Par ailleurs, c'est en définissant le milieu d'origine par le diplôme le plus élevé des deux parents que l'affaiblissement du lien est le moins prononcé : en rythme annuel, - 0,5\% pour l'ensemble et pour les hommes, $-0,6 \%$ pour les femmes, ce qui est toujours inférieur à la tendance observée avec chacune des autres manières d'approcher le milieu d'origine (au moins - 0,7\%). Dans la perspective réelle évoquée plus haut, cela peut s'interpréter comme l'indice d'une réduction des inégalités culturelles devant l'école moins prononcée que celle des inégalités purement socio-économiques (cf. graphique $\mathrm{X}$ ).

\section{Graphique X \\ Lien entre origine sociale et diplôme sous le modèle d'évolution uniforme des rapports de chances (1)}

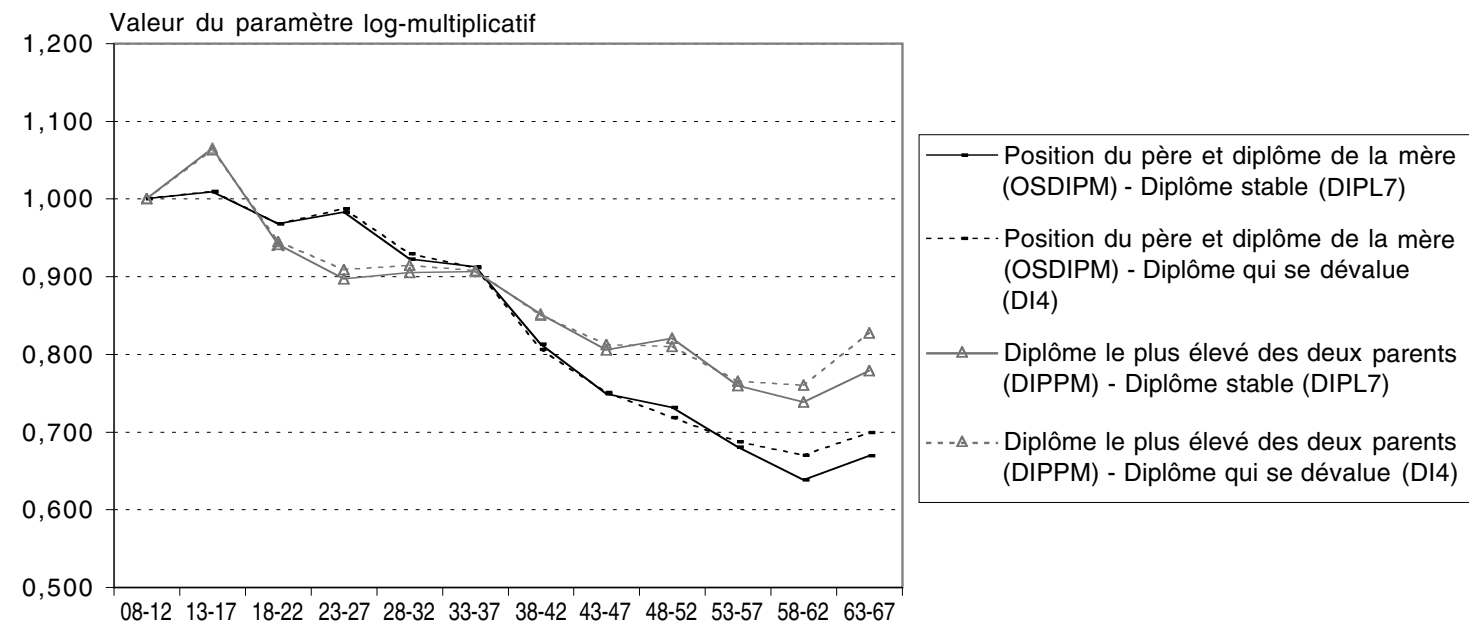

Génération quinquennale $19 \ldots$

1. L'échantillon considéré ici a un effectif total de 66781 individus. Pour le champ et la source, on se reportera à l'encadré 1. Lecture : de la génération 1908-1912 à la génération 1963-1967, le lien entre position du père/diplôme de la mère et diplôme s'est davantage affaibli que le lien entre diplôme le plus élevé des deux parents et diplôme. 
Si l'on retient la description du milieu d'origine la plus complète - la position du père, combinée avec le diplôme le plus élevé de la mère -, l'affaiblissement du lien entre milieu d'origine et diplôme, estimé dans le cadre du modèle 3 , suit un profil assez voisin de celui obtenu en considérant la seule position du père. Il est même semblable dans son rythme annuel moyen :- $0,8 \%$ pour l'ensemble, soit - 0,7 \% pour les hommes et $-0,9 \%$ pour les femmes (cf. graphique X). On aperçoit, en outre, à quel point le constat est analogue, que l'on suppose une stabilité ou une diminution de la valeur des diplômes.

Ce modèle, qui suppose une même évolution de tous les rapports des chances relatives, classe de manière erronée $4,6 \%$ des personnes. Cet écart non négligeable est réduit à 3,0\% lorsqu'on laisse les mêmes rapports libres d'évoluer dans des directions différentes au cours du temps (modèle 4). Avec ce modèle, la statistique de test est divisée par deux et, selon la génération, $66 \%$ à $74 \%$ des rapports des chances relatives se rapprochent de 1 et correspondent ainsi à une démocratisation qualitative effective. De nouveau, les tendances à une réduction des inégalités devant l'école ne se manifestent donc pas de façon uniforme, mais elles sont majoritaires.

\section{Revenir aux données}

Ainsi la démocratisation s'impose-t-elle comme une réalité tangible au regard des données, et indépendante du cadre statistique utilisé. Afin de préciser les conditions dans lesquelles elle s'exerce et à titre d'illustration, on s'intéresse pour finir aux destinées scolaires qu'ont connues, à 60 ans de distance, les enfants issus de quelques groupes sociaux, ainsi qu'à l'évolution du recrutement social des titulaires de certains diplômes. La robustesse des résultats attestée par ce qui précède permet de se limiter à un choix unique des nomenclatures: en ce qui concerne les diplômes, on suppose leur valeur stable, et le milieu d'origine est défini à partir de la position paternelle et du diplôme maternel le plus élevé. L'évolution des destinées scolaires ne sera plus appréhendée au travers du lien entre origine sociale et titre scolaire (force du lien global mesurée par le $V$ de Cramer, ou bien force évaluée en tant que paramètre du modèle 3), mais par de simples tables de destinées.

Le contraste entre les enfants d'ouvriers scolairement démunis (père ouvrier et mère sans diplôme) et ceux d'ouvriers scolairement pourvus (père ouvrier et mère diplômée, du CEP jusqu'au baccalauréat inclus) illustre bien le rôle du niveau culturel des parents (cf. tableau 4). On confirme que le niveau scolaire de la mère est un atout de taille. Dans la génération née entre 1963 et 1967, la destinée scolaire des enfants de ces deux segments du milieu ouvrier est nettement différente: un quart des enfants d'ouvriers scolairement démunis auraient dû acquérir un diplôme plus élevé pour que leur répartition soit identique à celle des enfants d'ouvriers scolairement pourvus. L'écart est donc important, mais il l'était encore davantage et dépassait un tiers parmi les enfants des familles ouvrières nés entre 1908 et 1912 (13).

13. On utilise ici l'indice de dissimilarité entre deux distributions, c'est-à-dire la demi-somme des valeurs absolues des différences de pourcentages.

\section{Tableau 4}

Diplôme des enfants d'ouvriers selon que leur mère a ou n'a pas de diplôme

\begin{tabular}{|c|c|c|c|c|c|c|c|c|c|}
\hline & & $\begin{array}{l}\text { Aucun } \\
\text { diplôme }\end{array}$ & CEP & CAP, BEP & $\begin{array}{l}\text { Brevet } \\
\text { BEPC }\end{array}$ & BAC & $\mathrm{BAC}+2$ & $\begin{array}{l}\mathrm{BAC}+3 \\
\text { et plus }\end{array}$ & Ensemble \\
\hline \multirow{2}{*}{$\begin{array}{l}\text { Génération } \\
1908-1912\end{array}$} & $\begin{array}{l}\text { Enfants de père ouvrier } \\
\text { et de mère sans diplôme }\end{array}$ & 65,5 & 27,2 & 3,3 & 1,7 & 1,7 & \multicolumn{2}{|c|}{0,5} & 100 \\
\hline & $\begin{array}{l}\text { Enfants de père ouvrier } \\
\text { et de mère diplômée }\end{array}$ & 30,9 & 54,8 & 6,3 & 5,7 & 0,8 & \multicolumn{2}{|c|}{1,5} & 100 \\
\hline \multirow{2}{*}{$\begin{array}{l}\text { Génération } \\
1963-1967\end{array}$} & $\begin{array}{l}\text { Enfants de père ouvrier } \\
\text { et de mère sans diplôme }\end{array}$ & \multicolumn{2}{|l|}{43,2} & 36,6 & 5,3 & 8,4 & 4,9 & 1,6 & 100 \\
\hline & $\begin{array}{l}\text { Enfants de père ouvrier } \\
\text { et de mère diplômée }\end{array}$ & \multicolumn{2}{|l|}{17,7} & 43,2 & 7,7 & 17,4 & 8,9 & 5,2 & 100 \\
\hline
\end{tabular}

Champ : individus français de naissance dont le père était ouvrier (y compris contremaître et ouvrier agricole) et la mère était sans diplôme (y compris non déclaré) ou bien diplômée, du CEP au baccalauréat inclus.

Source: enquête FQP de 1977 (recodification en PCS) pour la génération 1908-1912; enquête FQP de 1993 pour la génération 1963-1967. 
Par ailleurs, en 60 ans, les enfants d'ouvriers dont la mère est diplômée se sont un peu rapprochés des enfants de leur génération très avantagés scolairement (ceux qui sont issus de la famille d'un cadre supérieur ou d'un enseignant et dont la mère a au plus le baccalauréat serviront de référence) : l'indice de dissimilarité entre les deux distributions décline en effet de $50,4 \%$ à 42,0 \% (cf. tableau 5). À l'inverse, les enfants d'ouvriers dont la mère est sans diplôme se sont éloignés des enfants scolairement avantagés : le même indice enregistre en effet entre ces deux catégories un écart légèrement croissant (de 57,4 \% à 60,9 \%). De telles évolutions peuvent paraître contradictoires : sous l'angle des destinées scolaires de leurs enfants, les fractions diplômée et non diplômée du milieu ouvrier se seraient rapprochées, mais la première serait aujourd'hui moins éloignée des catégories supérieures alors que la seconde le serait davantage ! Cet exemple illustre la difficulté des comparaisons, dès lors qu'on les conduit à l'aide d'indices, certes simples a priori, mais imparfaits.

Le rôle du capital culturel de la famille peut encore être illustré à partir de l'exemple des enfants de paysans. On a conclu plus haut que leur réussite scolaire non seulement s'était élevée, mais qu'elle l'avait fait davantage que sous la seule poussée de l'allongement général des études. Ce progrès très sensible se voit principalement lorsque la famille paysanne d'origine est dotée d'un capital scolaire: quand leur mère est diplômée, les enfants d'agriculteurs des jeunes générations réussissent beaucoup mieux que leurs aînés. Par exemple, parmi ceux qui sont nés entre 1963 et 1967 et dont la mère était diplômée, près de la moitié ont obtenu au moins le baccalauréat. Presque tous ceux qui étaient nés avant la Première Guerre mondiale avaient au plus le certificat d'études : rien (48\%) ou ce diplôme seulement (40 \%). Parmi les enfants de la paysannerie dont la famille ne disposait pas de capital culturel, l'élévation du niveau de formation est également perceptible, mais il s'avère moins sensible.

On compare pour finir les titulaires d'un diplôme supérieur court et ceux d'un diplôme supérieur long. Avec l'allongement général des études, la possession d'un diplôme du supérieur a cessé d'être un privilège rare : $2 \%$ des personnes de la génération 1908-1912 étaient diplômées du supérieur, 33 \% de celles de la génération 1968-1972 (cf. tableau 1) (14). En conséquence, l'hétérogénéité des titulaires de diplômes supérieurs a augmenté, ce qui ressort du contraste entre les origines des diplômés du supérieur court (en y incluant les diplômes technologiques, BTS et DUT) et celles des diplômés du supérieur long (au moins la licence, concours de recrutement des professeurs, diplômés d'une grande école, docteurs, etc.).

Les seconds se distinguent en effet par leur origine sociale sensiblement plus favorisée (cf. tableau 6). D’ailleurs, les diplômés du supérieur court sont souvent, une fois sur trois, des

14. Si l'on abandonne l'étude par génération pour raisonner sur toute la population active à partir des recensements et enquêtes sur l'emploi, 2,1 \% des actifs avaient un diplôme supérieur au baccalauréat en 1954 contre 21,0 \% en 1996 (Marchand et Thélot, 1997).

Tableau 5

Carrières scolaires extrêmes : comparaison entre enfants « avantagés 》 et " désavantagés 》

En \%

\begin{tabular}{|c|c|c|c|c|c|c|c|c|c|}
\hline & & $\begin{array}{l}\text { Aucun } \\
\text { diplôme }\end{array}$ & CEP & $\begin{array}{l}\text { CAP } \\
\text { BEP }\end{array}$ & $\begin{array}{l}\text { Brevet } \\
\text { BEPC }\end{array}$ & BAC & $B A C+2$ & $\begin{array}{c}\text { BAC }+3 \\
\text { et plus }\end{array}$ & Ensemble \\
\hline Génération & Enfants «avantagés» & 13,3 & 22,0 & 13,0 & 12,8 & 17,5 & \multicolumn{2}{|c|}{21,4} & 100 \\
\hline $1908-1912$ & Enfants “désavantagés» & 65,5 & 27,2 & 3,3 & 1,7 & 1,7 & \multicolumn{2}{|c|}{0,5} & 100 \\
\hline & Ensemble de la génération & 51,5 & 32,7 & 6,2 & 3,8 & 3,4 & \multicolumn{2}{|c|}{2,4} & 100 \\
\hline Génération & Enfants «avantagés» & \multicolumn{2}{|c|}{6,9} & 12,0 & 7,7 & 18,5 & 27,3 & 27,6 & 100 \\
\hline $1963-1967$ & Enfants «désavantagés» & \multicolumn{2}{|c|}{43,2} & 36,6 & 5,3 & 8,4 & 4,9 & 1,6 & 100 \\
\hline & Ensemble de la génération & \multicolumn{2}{|c|}{20,7} & 33,4 & 8,4 & 15,7 & 12,0 & 9,8 & 100 \\
\hline
\end{tabular}

Lecture: Enfants "avantagés» : enfants dont le père est cadre supérieur, gros indépendant ou enseignant et la mère a au plus le baccalauréat ;

Enfants "désavantagés» : enfants dont le père est ouvrier (y compris contremaître et ouvrier agricole) et la mère n'a aucun diplôme (y compris non déclaré).

Les enfants "avantagés" considérés ici ne sont pas, en moyenne, les plus avantagés que notre nomenclature permettrait de définir. Ceux-ci sont les enfants dont la mère a un diplôme supérieur au baccalauréat et le père une position quelconque, mais leur effectif est trop faible dans la génération 1908-1912 pour qu'il soit possible de retenir cette catégorie.

Champ : individus français de naissance, ayant précisé la situation professionnelle de leur père.

Source : enquête FQP de 1977 (recodification en PCS) pour la génération 1908-1912; enquête FQP de 1993 pour la génération $1963-1967$. 


\begin{tabular}{|c|c|c|c|c|c|c|c|c|}
\hline & \multicolumn{6}{|c|}{$\begin{array}{c}\text { Principales origines sociales ... } \\
\text { Génération 1963-1967 }\end{array}$} & \multicolumn{2}{|c|}{ Génération 1908-1912 } \\
\hline & \multicolumn{2}{|c|}{$\begin{array}{l}\text {... des diplômés } \\
\text { du supérieur long }\end{array}$} & \multicolumn{2}{|c|}{$\begin{array}{l}\text {... des diplômés } \\
\text { du supérieur court }\end{array}$} & \multicolumn{2}{|c|}{$\begin{array}{l}\text {... des diplômés } \\
\text { du supérieur }\end{array}$} & \multicolumn{2}{|c|}{$\begin{array}{l}\text {... des diplômés } \\
\text { du supérieur }\end{array}$} \\
\hline $\begin{array}{l}\text { Père cadre supérieur, gros indépendant } \\
\text { ou enseignant et mère ayant au plus le bac }\end{array}$ & 31,7 & & & 25,4 & & 28,3 & 39,6 & \\
\hline Mère diplômée d'au moins bac +2 & 26,2 & 69,7 & & 10,9 & & 17,7 & 8,0 & \\
\hline $\begin{array}{l}\text { Père ouvrier et mère } \\
\text { diplômée du CEP au bac }\end{array}$ & 11,8 & & & 16,5 & & 14,4 & & \\
\hline $\begin{array}{l}\text { Père employé ou cadre moyen } \\
\text { et mère diplômée du CEP au bac }\end{array}$ & & & & 16,5 & & 13,4 & 14,0 & \\
\hline $\begin{array}{l}\text { Père employé ou cadre moyen } \\
\text { et mère sans diplôme }\end{array}$ & & & & & & & 8,6 & \\
\hline
\end{tabular}

Champ : individus français de naissance, ayant obtenu un diplôme supérieur au baccalauréat et ayant précisé la situation professionnelle de leur père.

Source : enquête FQP de 1977 (recodification en PCS) pour la génération 1908-1912 ; enquête FQP de 1993 pour la génération $1963-1967$.

enfants d'ouvriers ou de couches moyennes, dès lors que leur mère était elle-même diplômée. Les enfants d'ouvriers scolairement pourvus constituent désormais, pour l'enseignement supérieur, l'un des trois viviers les plus importants, ce qui n'était pas le cas auparavant. Il reste que près de la moitié des diplômés du supérieur continuent à venir des milieux favorisés (père cadre supérieur ou enseignant ou bien mère très diplômée) : 48 \% dans la génération 1908-1912, 46 \% dans la génération 1963-1967. L'origine sociale et scolaire de ces personnes ayant atteint les sommets du système éducatif illustre aussi bien la réduction des inégalités au cours du temps que l'existence, aujourd'hui encore, d'inégalités sensibles.

$$
\text { * * } *
$$

Ce sont d'ailleurs les deux conclusions générales qui se dégagent de cet article: les inégalités sociales devant l'école demeurent, aujourd'hui, fortes ; elles ont, cependant, diminué depuis quelques décennies, non seulement du fait de l'allongement général des études, mais aussi au-delà, de façon qualitative. Quelles interprétations principales donner à ces inégalités ? En particulier, s'agit-il d'inégalités liées à l'offre éducative, qui n'est pas la même partout (en campagne et en ville, dans les différents établissements, etc.) ou d'inégalités liées à la demande, c'est-à-dire à la position et au comportement des élèves ou des familles ? Les sociologues se sont souvent penchés sur cette question. Selon la réponse fournie, la politique qu'on décidera de conduire pour continuer à réduire les inégalités devant l'école - cette réduction est en effet un objectif assigné au système éducatif, inscrit en tant que tel dans la loi d'orientation de 1989 - différera.

Les auteurs remercient un rapporteur anonyme de ses remarques sur la version initiale de ce texte.

\section{BIBLIOGRAPHIE}

Agresti A. (1990), Categorical Data Analysis, New York, Wiley.

Baudelot C. (1989), «L'âge rend-il plus savant ? Un exemple de biais de réponse dans les enquêtes » in L'Hardy P. et Thélot C. (sous la direction de), Les Ménages. Mélanges en l'honneur de Jacques Desabie, Paris, Insee, pp. 159-173.
Baudelot C. et Establet R. (1992), Allez les filles !, Paris, Le Seuil.

Bishop Y.M.M., Fienberg S.E. et Holland P.W. (1975), Discrete Multivariate Analysis: Theory and Practice, Cambridge, MIT Press.

Brauns H. (1998), Bildung in Frankreich. Eine Studie zum Wandel herkunfts- und geschlechtsspezifischen Bildungsverhaltens, Opladen, Leske und Budrich. 
Coëffic N. (1998), « Parcours scolaires au collège et au lycée », Note d'information, 98-01, Ministère de l'Éducation nationale, de la Recherche et de la Technologie, Direction de la Programmation et du Développement.

Degenne An., Lebeaux M.-O. et Vallet L.-A. (1998), Les données de l'enquête Formation/ Qualification Professionnelle de 1964 sont de nouveau disponibles, LASMAS-Institut du Longitudinal (CNRS), 163 p.

Duru-Bellat M. (1994 et 1995), « Filles et garçons à l'école, approches sociologiques et psychosociales », Revue française de pédagogie, 109, pp. 111-141 et 110 , pp. 75-109.

Duru-Bellat M. et Kieffer A. (1999), « La démocratisation de l'enseignement « revisitée ». Une mise en perspective historique et internationale des inégalités des chances scolaires en France », Les Cahiers de l'Irédu, n 60.

Erikson R. et Goldthorpe J.H. (1992), The Constant Flux. A Study of Class Mobility in Industrial Societies, Oxford, Clarendon Press.

Garnier M.A. et Raffalovich L.E. (1984), « The Evolution of Equality of Educational Opportunities in France », Sociology of Education, 57(1), pp. 1-11.

Goodman L.A. et Hout M. (1998), « Statistical Methods and Graphical Displays for Analyzing how the Association Between Two Qualitative Variables Differs among Countries, among Groups, or Over Time: a Modified RegressionType Approach », Sociological Methodology, 28, pp. $175-230$.

Goux D. et Maurin É. (1995), « Origine sociale et destinée scolaire. L'inégalité des chances devant l'enseignement à travers les enquêtes Formation Qualification Professionnelle 1970, 1977, 1985 et 1993 », Revue française de sociologie, 36 (1), pp. 81-121.

Goux D. et Maurin É. (1997), «Démocratisation de l'école et persistance des inégalités », Économie et Statistique, 306, pp. 27-39.

Jonsson J.O., Mills C. et Müller W. (1996), «A Half Century of Increasing Educational
Openness? Social Class, Gender and Educational Attainment in Sweden, Germany and Britain » in Erikson R. et Jonsson J.O. (eds.), Can Education Be Equalized? The Swedish Case in Comparative Perspective, Boulder, Westview Press, pp. 183-206.

Joutard P. et Thélot C. (1999), Réussir l'École. Pour une politique éducative, Paris, Le Seuil.

Marchand O. et Thélot C. (1997), Le Travail en France (1800-2000), Paris, Nathan.

Mare R.D. (1980), « Social Background and School Continuation Decisions », Journal of the American Statistical Association, 75, pp. 295-305.

Mare R.D. (1981), «Change and Stability in Educational Stratification », American Sociological Review, 46(1), pp. 72-87.

Powers D.A. et Xie Y. (2000), Statistical Methods for Categorical Data Analysis, San Diego, Academic Press.

Prost A. (1986), L'enseignement s'est-il démocratisé ? Les élèves des lycées et collèges de l'agglomération d'Orléans de 1945 à 1980, Paris, Presses Universitaires de France, ( $2^{\mathrm{e}}$ éd. revue et augmentée, 1992).

Raftery A.E. (1995), « Bayesian Model Selection in Social Research », Sociological Methodology, 25, pp. 111-163.

Smith H.L. et Garnier M.A. (1986), «Association Between Background and Educational Attainment in France », Sociological Methods \& Research, 14(3), pp. 317-344.

Vallet L.-A. (1999), « Quarante années de mobilité sociale en France. L'évolution de la fluidité sociale à la lumière de modèles récents ", Revue française de sociologie, 40(1), pp. 5-64.

Vermunt J.K. (1997), LEM: A general program for the analysis of categorical data, Version 1.0, Tilburg University.

Xie Y. (1992), «The Log-Multiplicative Layer Effect Model for Comparing Mobility Tables », American Sociological Review, 57(3), pp. 380-395. 